\title{
Eigenvalue Clusters of Large Tetradiagonal Toeplitz Matrices
}

\author{
Albrecht Böttcher®, Juanita Gasca, Sergei M. Grudsky and \\ Anatoli V. Kozak
}

\begin{abstract}
Toeplitz matrices are typically non-Hermitian and hence they evade the well-elaborated machinery one can employ in the Hermitian case. In a pioneering paper of 1960, Palle Schmidt and Frank Spitzer showed that the eigenvalues of large banded Toeplitz matrices cluster along a certain limiting set which is the union of finitely many closed analytic arcs. Finding this limiting set nevertheless remains a challenge. We here present an algorithm in the spirit of Richard Beam and Robert Warming that reduces testing $O\left(N^{2}\right)$ points in the plane for membership in the limiting set by testing only $O(N)$ points along a one-dimensional curve. For tetradiagonal Toeplitz matrices, we describe all types of the limiting sets, we classify their exceptional points, and we establish asymptotic formulas for the analytic arcs near their endpoints.
\end{abstract}

Mathematics Subject Classification. Primary 47B35; Secondary 15A18, 15B05, 65F15.

Keywords. Toeplitz matrix, Tetradiagonal matrix, Eigenvalue cluster, Limiting set.

\section{Introduction}

It is well known that the properties of truncated $n \times n$ Toeplitz matrices

$$
\left(a_{j-k}\right)_{j, k=1}^{n}=\left(\begin{array}{cccc}
a_{0} & a_{-1} & \ldots & a_{-(n-1)} \\
a_{1} & a_{0} & \ldots & a_{-(n-2)} \\
\vdots & \vdots & \ddots & \vdots \\
a_{n-1} & a_{n-2} & \ldots & a_{0}
\end{array}\right)
$$

as $n$ goes to infinity are captured by the series $a(z)=\sum_{j=-\infty}^{\infty} a_{j} z^{j}(z \in$ $\mathbb{C})$. We therefore denote the matrix $(1.1)$ by $T_{n}(a)$. The first Szegö limit theorem [12] describes the asymptotic eigenvalue distribution of $T_{n}(a)$ in the case where the restriction of $a$ to the complex unit circle $\mathbb{T}$ is (the Fourier 
series of) a real-valued $L^{\infty}$ function. More recent studies extend this to realvalued $L^{1}$ functions and even more general Hermitian situations; see, e.g., [6, 14-16]. The problem is of a completely different nature if the restriction $a \mid \mathbb{T}$ is complex-valued. Here the path-breaking result is due to the 1960 paper [10] by Schmidt and Spitzer.

Thus, let us consider the banded Toeplitz matrix

$$
\left(\begin{array}{lllll}
a_{0} & \ldots & a_{-r} & & \\
\vdots & \ddots & & \ddots & \\
a_{s} & & a_{0} & & a_{-r} \\
& \ddots & & \ddots & \vdots \\
& & a_{s} & \ldots & a_{0}
\end{array}\right)_{n \times n} .
$$

Throughout what follows we always assume that $a_{j} \in \mathbb{C}, r \geq 1, s \geq 1$, $a_{-r} \neq 0$, and $a_{s} \neq 0$. Schmidt and Spitzer [10] showed that the set $\sigma\left(T_{n}(a)\right)$ of the eigenvalues of $T_{n}(a)$ converges in the Hausdorff metric to some limiting set $\Lambda(a)$ which is the union of finitely many analytic arcs and their endpoints and which does not contain isolated points. Ullman [17] later proved that $\Lambda(a)$ is connected.

It is tempting to say that once we know that the limiting set exists, we could simply compute the eigenvalues of $T_{n}(a)$ for $n=100$ and this should give a good approximation to $\Lambda(a)$. Well, let

$$
a(z)=z^{3}-3(1+i) z^{2}+7 i z+4(1-i)-2 z^{-1} .
$$

We thus have a pentadiagonal matrix. Figure 1a shows the curve $a(\mathbb{T})$ and the eigenvalues of $T_{100}(a)$ delivered by Matlab. Clearly, part of the eigenvalues are erroneous. Even after zooming in it is impossible to guess what $\Lambda(a)$ is.

Already Schmidt and Spitzer employed the key trick with banded Toeplitz matrices: namely, if $D_{\varrho}=\operatorname{diag}\left(\varrho^{j-1}\right)_{j=1}^{n}$, then

$$
D_{\varrho}\left(a_{j-k}\right)_{j, k=1}^{n} D_{\varrho}^{-1}=\left(\varrho^{j-k} a_{j-k}\right)_{j, k=1}^{n}
$$

and hence $T_{n}(a)=\left(a_{j-k}\right)_{j, k=1}^{n}$ and $\left(\varrho^{j-k} a_{j-k}\right)_{j, k=1}^{n}$ have the same eigenvalues. The last matrix may be written as $T_{n}\left(a_{\varrho}\right)$ with $a_{\varrho}(z)=\sum_{j=-r}^{s} \varrho^{j} a_{j} z^{j}$. Figure $1 \mathrm{~b}-\mathrm{d}$ shows $a_{\varrho}(\mathbb{T})$ and the (in part erroneous) eigenvalues of $T_{100}\left(a_{\varrho}\right)$ computed with Matlab for three choices of the parameter $\varrho$. Without knowing more on $\Lambda(a)$ it is difficult to make a reliable guess.

Let us turn to the tetradiagonal case. A subset of $\mathbb{C}$ consisting of two distinct points $\mu, \nu \in \mathbb{C}$ and an analytic arc (without self-intersection) joining these two points will be denoted by $[\mu \sim \nu]$. Part of our main result is as follows.

Let $\gamma$ be the piece of the curve $\left\{b \in \mathbb{C}:|1+b|=2|b|^{2}\right\}$ that lies in the closed disk $\{b \in \mathbb{C}:|1+b| \leq 1\}$ (see Fig. 7) and let $\Gamma$ be the curve

$$
\Gamma=\left\{ \pm 2 \frac{\left(1+b+b^{2}\right)^{3 / 2}}{b(1+b)}: b \in \gamma\right\} \text {. }
$$




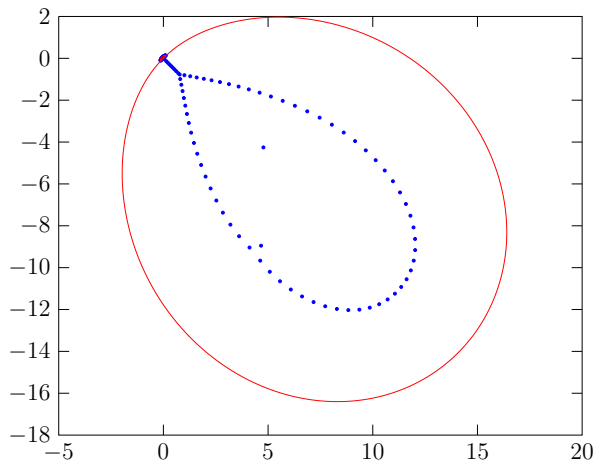

(a) $\varrho=1, n=100$

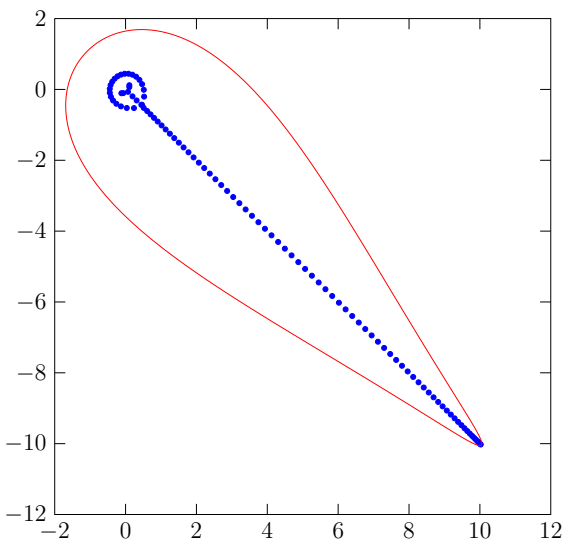

(c) $\varrho=0.4, n=100$

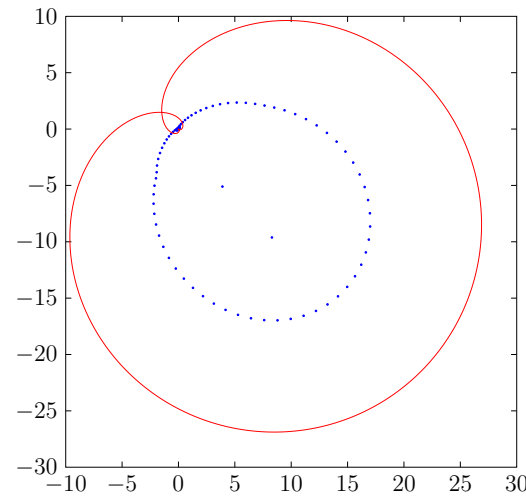

(b) $\varrho=1.5, n=100$

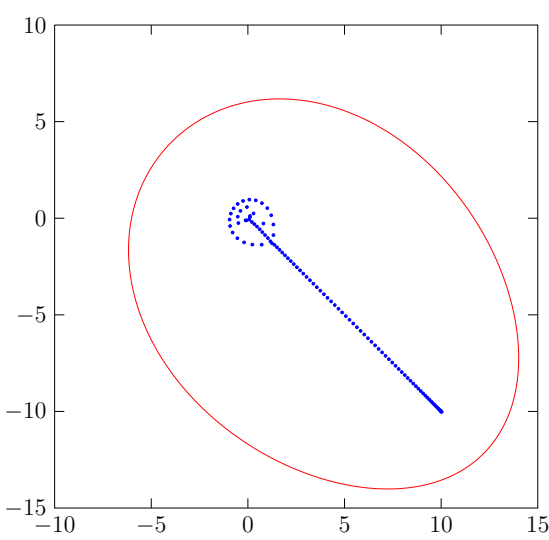

(d) $\varrho=0.2, n=100$

FiguRE 1. What is the limiting set?

The \pm indicates that we take both values of $\left(1+b+b^{2}\right)^{3 / 2}$. The curve $\Gamma$ is the boundary of the blue domain $\Omega$ shown in Fig. 2. The two red points are $\pm 3 \sqrt{3}$ and they correspond to $b=-1 / 2 \in \gamma$.

As will be seen below, the key trick allows us to assume without loss of generality that $a(z)=z^{2}+c z+c z^{-1}$ with $c \in \mathbb{C} \backslash\{0\}$. Denote the roots of $a^{\prime}(z)=0$ by $t_{1}, t_{2}, t_{3}$ and order them so that $\left|t_{3}\right| \leq\left|t_{2}\right| \leq\left|t_{1}\right|$. Put $\lambda_{k}=a\left(t_{k}\right)$. Then $\Lambda(a)$ is a set of one of the following types. If $c$ is in the interior of $\Omega$, then the three points $\lambda_{1}, \lambda_{2}, \lambda_{3}$ are distinct and

$$
\Lambda(a)=\left[\lambda_{0} \sim \lambda_{1}\right] \cup\left[\lambda_{0} \sim \lambda_{2}\right] \cup\left[\lambda_{0} \sim \lambda_{3}\right] \quad \text { with } \quad \lambda_{0}=-|c|^{4 / 3} ;
$$

the three arcs intersect only in $\lambda_{0}$. If $c$ lies on the boundary of the set $\Omega$, then $\Lambda(a)=\left[\lambda_{1} \sim \lambda_{2}\right] \cup\left[\lambda_{1} \sim \lambda_{3}\right]$; if $c= \pm 3 \sqrt{3}$, then $\lambda_{1}=\lambda_{2} \neq \lambda_{3}$ and $\Lambda(a)$ is actually the straight line segment $\left[\lambda_{2}, \lambda_{3}\right]$, while if $c \neq \pm 3 \sqrt{3}$, the three points $\lambda_{1}, \lambda_{2}, \lambda_{3}$ are distinct and the two arcs intersect only in $\lambda_{1}$, making there a cusp. Finally, if $c$ is outside the closed set $\Omega$, then $\lambda_{2} \neq \lambda_{3}$ and 


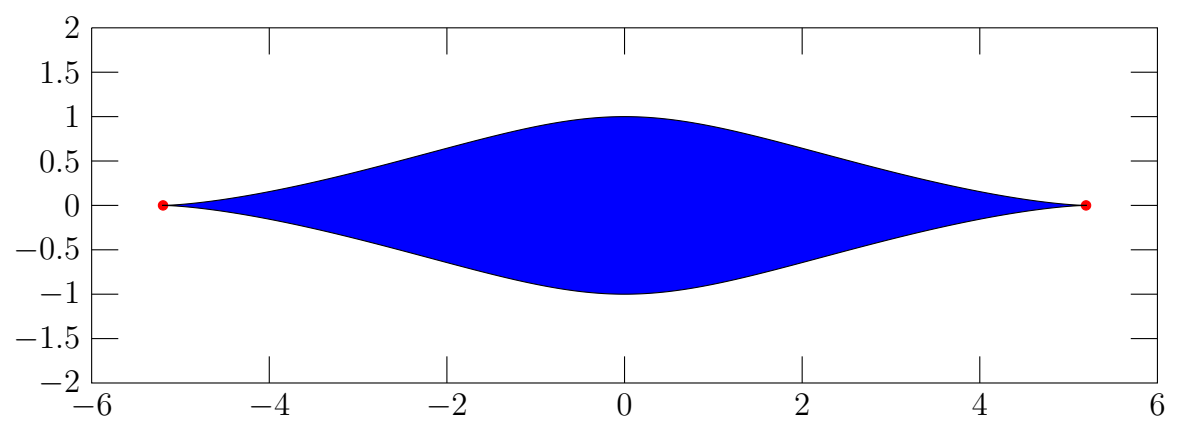

Figure 2. The set $\Omega$

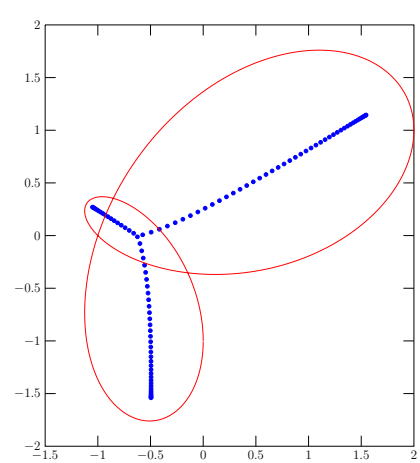

(a) $c=1 / 2+i / 2 \in \operatorname{int} \Omega, n=60$ Type $L_{3}$

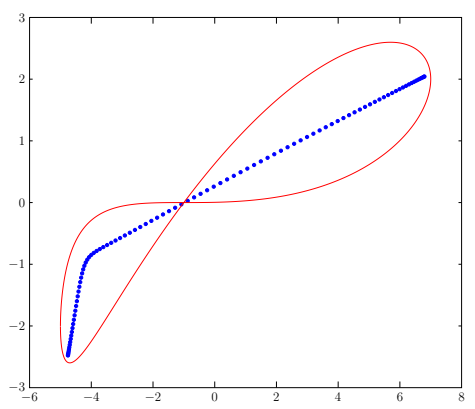

(c) $c=3+i \in \mathbb{C} \backslash \Omega, n=60$ Type $L_{1}$

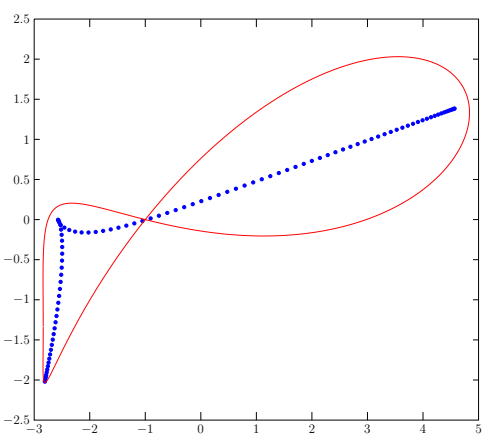

(b) $c=1.9187+0.6656 i \in \partial \Omega, n=60$ Type $L_{2}$

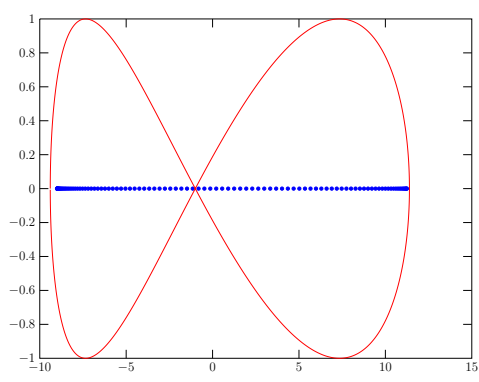

(d) $c=3 \sqrt{3} \approx 5.1962 \in \partial \Omega, n=60$ Degeneration

Figure 3. The four types of the limiting sets

$\Lambda(a)=\left[\lambda_{2} \sim \lambda_{3}\right]$. Figure 3 shows some examples along with the eigenvalues of $T_{60}(a)$. 
Schmidt and Spitzer [10] gave two descriptions of the limiting set $\Lambda(a)$. First, they showed that

$$
\Lambda(a)=\bigcap_{\varrho \in(0, \infty)} \sigma\left(T\left(a_{\varrho}\right)\right),
$$

where $\sigma\left(T\left(a_{\varrho}\right)\right)$ is the spectrum of the Toeplitz operator generated by the infinite Toeplitz matrix $\left(\varrho^{j-k} a_{j-k}\right)_{j, k=1}^{\infty}$ on $\ell^{2}$. Note that $\sigma\left(T\left(a_{\varrho}\right)\right)$ is just the union of the curve $a_{\varrho}(\mathbb{T})$ and the points in the plane about which this curve has nonzero winding number. For the second description, pick $\lambda \in \mathbb{C}$, consider the equation $a(z)=\lambda$ (which after multiplication by $z^{r}$ becomes an equation with an algebraic polynomial of degree $r+s)$, and order the solutions $z_{j}(\lambda)$ $(j=1, \ldots, r+s)$ of this equation so that

$$
\left|z_{1}(\lambda)\right| \leq\left|z_{2}(\lambda)\right| \leq \cdots \leq\left|z_{r+s}(\lambda)\right|
$$

Then $\lambda \in \Lambda(a)$ if and only if $\left|z_{r}(\lambda)\right|=\left|z_{r+1}(\lambda)\right|$.

The original reasoning of Schmidt and Spitzer was significantly simplified and accompanied with the density function for the eigenvalues along $\Lambda(a)$ by Hirschman [7] and Widom [18,19]; see also Chapter 11 of [2]. A new view at the matter is given by Duits and Kuijlaars in [5]. We should also note that in the tridiagonal case $a(z)=a_{1} z+a_{0}+a_{-1} z^{-1}$ we have

$$
\Lambda(a)=\left[a_{0}-2 \sqrt{a_{1} a_{-1}}, a_{0}+2 \sqrt{a_{1} a_{-1}}\right] .
$$

If $a(z)=\sum_{j=-r}^{s} a_{j} z^{j}$ takes only real values on $\mathbb{T}$, then

$$
\Lambda(a)=[\min a(\mathbb{T}), \max a(\mathbb{T})] .
$$

Recently, Shapiro and Štampach [11] very beautifully characterized the Laurent polynomials for which $\Lambda(a)$ is a subset of the real line $\mathbb{R}$ : this happens if and only if the pre-image $a^{-1}(\mathbb{R})$ contains a Jordan curve. We refer to the books $[2,3,6,8]$ for more on the eigenvalue cluster problem for Toeplitz matrices. An analogue of the Schmidt-Spitzer result for Wiener-Hopf integral operators is in [4]. We also want to mention the message of $[9,13]$ according to which in certain problems the eigenvalues of non-normal operators may drastically mislead us.

Finally, recall that $T_{n}(a)$ and $T_{n}\left(a_{\varrho}\right)$ have the same eigenvalues. It follows in particular that

$$
\sigma\left(T_{n}\left(a_{2} z^{2}+a_{1} z+a_{0}+a_{-1} z^{-1}\right)\right)=\sigma\left(T_{n}\left(\varrho^{2} a_{2} z^{2}+\varrho a_{1} z+a_{0}+\varrho^{-1} a_{-1} z^{-1}\right)\right) .
$$

If $a_{1}=0$, this reads

$$
\sigma\left(T_{n}\left(a_{2} z^{2}+a_{0}+a_{-1} z^{-1}\right)\right)=\sigma\left(T_{n}\left(\varrho^{2} a_{2} z^{2}+a_{0}+\varrho^{-1} a_{-1} z^{-1}\right)\right)
$$

and taking $\varrho$ so that $\varrho^{2} a_{2}=\varrho^{-1} a_{-1}$, we obtain that

$$
\sigma\left(T_{n}(a)\right)=\sigma\left(T_{n}\left(a_{2} z^{2}+a_{0}+a_{-1} z^{-1}\right)\right)=a_{0}+\varrho^{2} a_{2} \sigma\left(T_{n}\left(z^{2}+z^{-1}\right)\right),
$$

which leads to a concrete single case. If $a_{1} \neq 0$, we choose $\varrho$ so that $\varrho a_{1}=$ $\varrho^{-1} a_{-1}$ and get

$$
\sigma\left(T_{n}(a)\right)=\sigma\left(T_{n}\left(\varrho^{2} a_{2} z^{2}+\varrho a_{1} z+a_{0}+\varrho^{-1} a_{-1} z^{-1}\right)\right)
$$




$$
=a_{0}+\varrho^{2} a_{2} \sigma\left(T_{n}\left(z^{2}+c z+c z^{-1}\right)\right)
$$

with $c=\varrho a_{1} /\left(\varrho^{2} a_{2}\right)=\varrho^{-1} a_{-1} /\left(\varrho^{2} a_{2}\right)$. The limiting set $\Lambda\left(z^{2}+z^{-1}\right)$ was already determined by Schmidt and Spitzer: it is the star-shaped set

$$
\Lambda\left(z^{2}+z^{-1}\right)=\bigcup_{k=0,1,2} \varepsilon^{k}\left[0, \frac{3}{2} \sqrt[3]{2}\right] \quad \text { with } \quad \varepsilon=e^{2 \pi i / 3} .
$$

Thus, we are left with the one-parameter family $T_{n}\left(z^{2}+c z+c z^{-1}\right), c \in \mathbb{C} \backslash\{0\}$.

\section{An Algorithm for Calculating the Limiting Set}

The algorithm presented here is based on looking at the algorithm designed by Beam and Warming in [1] from a different perspective.

A point $\lambda$ is in $\Lambda(a)$ if and only if $\lambda=a\left(z_{r+1}(\lambda)\right)=a\left(z_{r}(\lambda)\right)$ holds with $z_{r+1}(\lambda) / z_{r}(\lambda)=e^{i \varphi}$ for some $\varphi \in(-\pi, \pi]$. Thus, the point $z_{r}(\lambda)$ satisfies the two equations $a(z)=\lambda$ and $a\left(e^{i \varphi} z\right)=\lambda$ and consequently, it is a root of the equation

$$
a(z)-a\left(e^{i \varphi} z\right)=0
$$

which is only of use for $\varphi \neq 0$. Instead of considering $a(z)=\lambda$ for $\lambda$ in the two-dimensional plane $\mathbb{C}$, we take $\varphi$ from the one-dimensional set $(-\pi, \pi] \backslash\{0\}$ and solve Eq. (2.1). Let $u_{k}(\varphi)(k=1, \ldots, r+s)$ be the solutions of this equation. For each $k$, we put $\lambda_{k}(\varphi)=a\left(u_{k}(\varphi)\right)$ and label the roots $z_{j, k}(\varphi)$ $(j=1, \ldots, r+s)$ of the equation $a(z)=\lambda_{k}(\varphi)$ so that

$$
\left|z_{1, k}(\lambda)\right| \leq\left|z_{2, k}(\lambda)\right| \leq \cdots \leq\left|z_{r+s, k}(\lambda)\right| \text {. }
$$

If $\left|z_{r+1, k}(\varphi)\right|=\left|z_{r, k}(\varphi)\right|$, then $\lambda_{k}(\varphi) \in \Lambda(a)$, and otherwise $\lambda_{k}(\varphi) \notin \Lambda(a)$. To tackle the remaining case $\varphi=0$, we replace Eq. (2.1) by

$$
\lim _{\varphi \rightarrow 0}\left(a(z)-a\left(e^{i \varphi} z\right)\right) / \varphi=0,
$$

that is, by $a^{\prime}(z)=0$. Let $t_{k}(k=1, \ldots, r+s)$ be the solutions of this equation and put $\lambda_{k}(0)=a\left(t_{k}\right)$ for each $k$. Then solve $a(z)=\lambda_{k}(0)$ for each $k$ and sort the roots $z_{j, k}(0)$ so that

$$
\left|z_{1, k}(0)\right| \leq\left|z_{2, k}(0)\right| \leq \cdots \leq\left|z_{r+s, k}(0)\right| .
$$

What results is that $\lambda_{k}(0) \in \Lambda(a)$ if $\left|z_{r+1, k}(0)\right|=\left|z_{r, k}(0)\right|$, while $\lambda_{k}(0) \notin \Lambda(a)$ otherwise.

In summary, instead of finding the zeros of a polynomial of degree $r+s$ parametrized by $\lambda$ on a grid of order $O\left(N^{2}\right)$ covering a bounded subset of $\mathbb{C}$ (one may take the convex hull of the range $a(\mathbb{T})$ or even better some $\left.\sigma\left(T\left(a_{\varrho}\right)\right)\right)$, we have to find the zeros of $r+s+1$ polynomials of degree $r+s$ parametrized by $\varphi \in(-\pi, \pi]$ and thus by points of a grid of order $O(N)$.

If $u$ satisfies $a(u)-a\left(e^{i \varphi} u\right)=0$, then $v=e^{i \varphi} u$ satisfies the equation $a(v)-a\left(e^{-i \varphi} v\right)=0$. Thus, the solutions $u_{1}(\varphi), \ldots, u_{r+s}(\varphi)$ coincide with the solutions $u_{1}(-\varphi), \ldots, u_{r+s}(-\varphi)$, which implies that after appropriate sorting we get $\lambda_{k}(\varphi)=\lambda_{k}(-\varphi)$ for all $k$. We therefore may restrict the algorithm to $\varphi \in[0, \pi]$. 
Example 2.1. Figure 4 illustrates the algorithm for the septadiagonal matrix $T_{n}(a)$ induced by

$$
a(z)=-i z^{3}+2 z^{2}+z+i z^{-1}+2 z^{-2}-z^{-3} .
$$

Example 2.2. We take the pentadiagonal matrix generated by the polynomial (1.2). Recall that the numerically obtained eigenvalues are in Fig. 1. The algorithm applied to $a_{\varrho}$ with $\varrho=0.4$ yields Fig. 5 . In the left picture, the numerically computed eigenvalues of $T_{100}\left(a_{\varrho}\right)$ are shown as blue circles and the limiting set computed with the algorithm is in green. The right picture is a zoom in of the left.

\section{The Different Points of the Limiting Set}

A point $\lambda \in \Lambda(a)$ is said to be a regular point if it has an open neighborhood $V \subset \mathbb{C}$ such that $\Lambda(a) \cap V$ is an analytic arc (without self-intersection) starting and ending at the boundary of $V$, and a point $\lambda \in \Lambda(a)$ is called an exceptional point if it is not regular. This is a purely (differential-)topological classification of the points of $\Lambda(a)$. As part of an analytic classification of the points of $\Lambda(a)$ we call a point $\lambda \in \Lambda(a)$ a simple point if

$$
\left|z_{r-1}(\lambda)\right|<\left|z_{r}(\lambda)\right|=\left|z_{r+1}(\lambda)\right|<\left|z_{r+2}(\lambda)\right| \quad \text { and } \quad z_{r}(\lambda) \neq z_{r+1}(\lambda) .
$$

Example 3.1. (A simple point need not be regular.) Consider the pentadiagonal case given by (1.2) and studied in Example 2.2. Straightforward computation shows that we have the factorization

$$
a(z)=(z-1)(z-i)(z-1-i)^{2} / z .
$$

The origin $\lambda=0$ belongs to $\Lambda(a)$ if and only if $\left|z_{1}(0)\right|=\left|z_{2}(0)\right|$. The solutions of $a(z)=0$ are

$$
z_{1}(0)=1, \quad z_{2}(0)=i, \quad z_{3}(0)=1+i, \quad z_{4}(0)=1+i .
$$

Thus, the point $\lambda=0$ is simple and $z_{2}(0) / z_{1}(0)=e^{i \varphi_{0}}$ with $\varphi_{0}=\pi / 2$. If $z=u(\varphi)$ is a solution of the equation

$$
\Phi(z, \varphi):=a(z)-a\left(e^{i \varphi} z\right)=0
$$

satisfying $u(\pi / 2)=1$, then $\lambda(\varphi)=a(u(\varphi))$ remains a simple point on $\Lambda(a)$ for $\varphi$ sufficiently close to $\pi / 2$. We have

$$
\Phi_{z}(z, \varphi)=a^{\prime}(z)-e^{i \varphi} a^{\prime}\left(e^{i \varphi} z\right), \quad \Phi_{z z}(z, \varphi)=a^{\prime \prime}(z)-e^{2 i \varphi} a^{\prime \prime}\left(e^{i \varphi} z\right) .
$$

From the representation (1.2) we get

$$
\Phi_{z}(1, \pi / 2)=a^{\prime}(1)-i a^{\prime}(i)=0, \quad \Phi_{z z}(1, \pi / 2)=a^{\prime \prime}(1)+a^{\prime \prime}(i)=-10(1+i) .
$$

Since $\Phi_{z}(1, \pi / 2)=0$ but $\Phi_{z z}(1, \pi / 2) \neq 0$, there is an open neighborhood $U \subset$ $\mathbb{C}$ of $\lambda=0$ such that $\Lambda(a) \cap U$ is formed by two analytic arcs perpendicularly intersecting in $\lambda=0$. Thus, $\lambda$ is not regular. The perpendicularly intersecting arcs are manifestly seen in Fig. 5 b. 


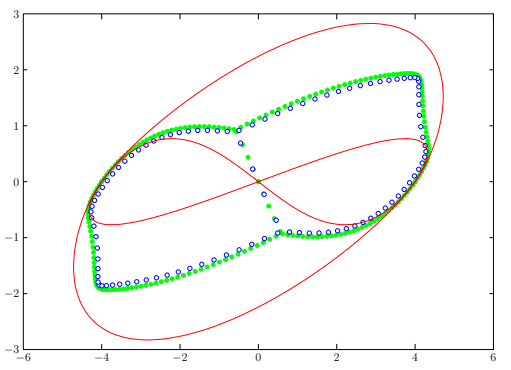

(a)

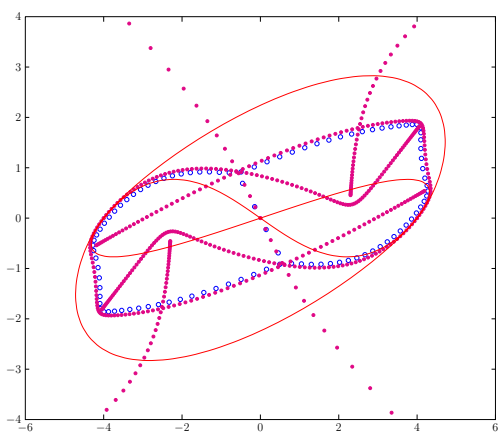

(c)

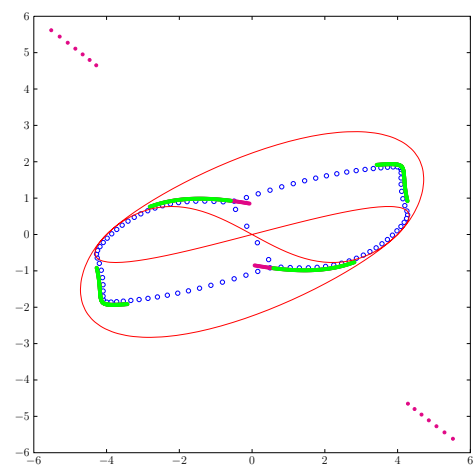

(e)

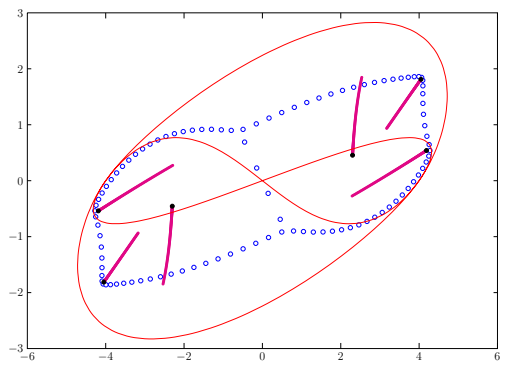

(b)

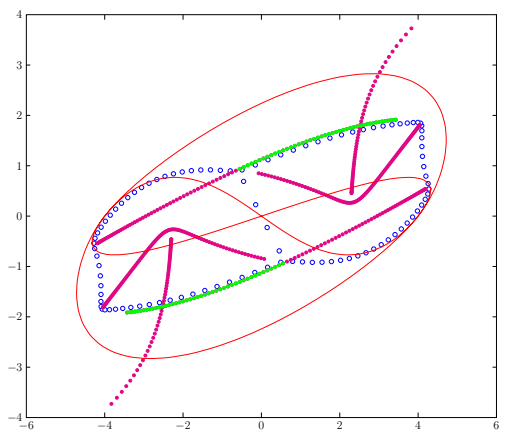

(d)

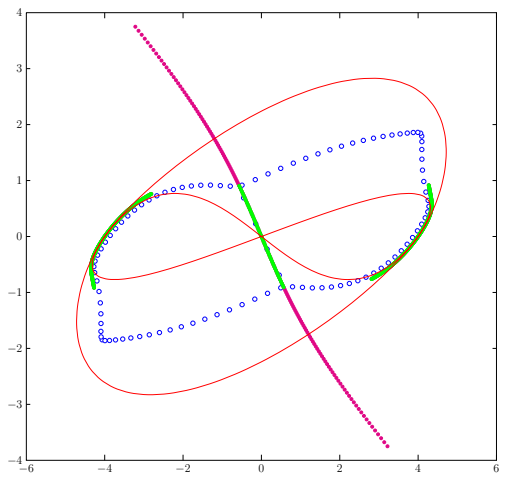

(f)

Figure 4 . In a we see the range $a(\mathbb{T})$ (red), the result of the algorithm for $\varphi=0.1: 0.1: \pi$ (green), and the eigenvalues of $T_{60}(a)$ (blue circles). b shows $\lambda_{1}(\varphi), \ldots, \lambda_{6}(\varphi)$ for $\varphi=$ $0.1: 0.01: 0.8$ (magenta). The black dots are the six values for $\varphi=0.1$. $\mathrm{c}$ This is $\lambda_{1}(\varphi), \ldots, \lambda_{6}(\varphi)$ for $\varphi=0.01: 0.01$ : $\pi$. In $\mathbf{d}$ we have $\lambda_{1}(\varphi), \ldots, \lambda_{6}(\varphi)$ for $\varphi=0.01: 0.01: 2$ (magenta) and the parts of these curves that form a piece of $\Lambda(a)$ (green). $\mathbf{e}$ is the same with $\lambda_{1}(\varphi), \ldots, \lambda_{6}(\varphi)$ for $\varphi=$ $2: 0.01: 2.7$, and $\mathbf{f}$ is the same with $\lambda_{1}(\varphi), \ldots, \lambda_{6}(\varphi)$ for $\varphi=2.7: 0.001: \pi$ 


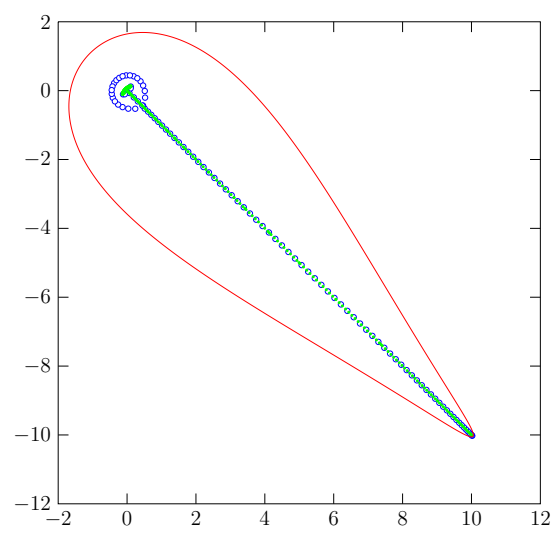

(a)

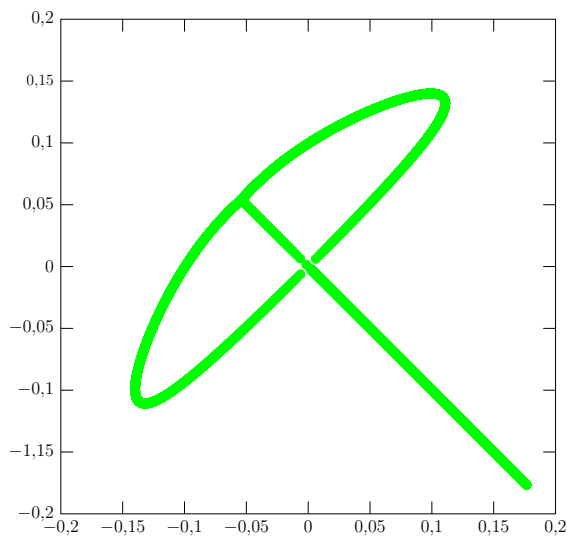

(b)

Figure 5. The true limiting set for the matrices of Fig. 1

If a point $\lambda \in \Lambda(a)$ is simple, that is, if it satisfies (3.1), then there is a unique number $\varphi \in(-\pi, \pi] \backslash\{0\}$ such that $z_{r+1}(\lambda) / z_{r}(\lambda)=e^{i \varphi}$. We say that the simple point $\lambda$ is nondegenerate if

$$
a^{\prime}\left(z_{r}(\lambda)\right)-e^{i \varphi} a^{\prime}\left(e^{i \varphi} z_{r}(\lambda)\right) \neq 0,
$$

and otherwise we call it degenerate. The point $\lambda=0$ in Example 3.1 was degenerate.

Theorem 3.2. A nondegenerate simple point is a regular point of $\Lambda(a)$.

Proof. Let $\lambda_{0} \in \Lambda(a)$ be a simple point with

$$
\left|z_{r-1}\left(\lambda_{0}\right)\right|<\left|z_{r}\left(\lambda_{0}\right)\right|=\left|z_{r+1}\left(\lambda_{0}\right)\right|<\left|z_{r+2}\left(\lambda_{0}\right)\right|
$$

and $z_{r}\left(\lambda_{0}\right) \neq z_{r+1}\left(\lambda_{0}\right)$. Then $z_{r+1}\left(\lambda_{0}\right) / z_{r}\left(\lambda_{0}\right)=e^{i \varphi_{0}}$ with $\varphi_{0} \in(-\pi, \pi] \backslash\{0\}$. We consider the equation $\Phi(u, \varphi):=a(u)-a\left(e^{i \varphi} u\right)=0$ with the initial condition $u\left(\varphi_{0}\right)=z_{r}\left(\lambda_{0}\right)=: u_{0}$. If $(\partial \Phi / \partial u)\left(u_{0}, \varphi_{0}\right) \neq 0$, the implicit function theorem ensures the existence of a unique analytic solution $u=u(\varphi)$ in a sufficiently small open neighborhood $V$ of $\varphi_{0}$ satisfying $u\left(\varphi_{0}\right)=u_{0}$. It follows that $\lambda(\varphi)=a(u(\varphi))$ describes an analytic arc in the plane. If $V$ is sufficiently small, we infer from (3.1) that

$$
\left|z_{r-1}(\lambda)\right|<\left|z_{r}(\lambda)\right|=\left|z_{r+1}(\lambda)\right|<\left|z_{r+2}(\lambda)\right|
$$

which implies that the arc is part of $\Lambda(a)$. Finally, the condition

$$
(\partial \Phi / \partial u)\left(u_{0}, \varphi_{0}\right) \neq 0
$$

is nothing but the condition

$$
a^{\prime}\left(u_{0}\right)-e^{i \varphi_{0}} a^{\prime}\left(e^{i \varphi_{0}} u_{0}\right)=a^{\prime}\left(z_{r}\left(\lambda_{0}\right)\right)-e^{i \varphi_{0}} a^{\prime}\left(e^{i \varphi_{0}} z_{r}\left(\lambda_{0}\right)\right) \neq 0,
$$

which in turn is just the nondegeneracy required.

Theorem 3.3. In the tetradiagonal case all simple points are nondegenerate and hence regular. 
Proof. Let $a(z)=a_{2} z^{2}+a_{1} z+a_{0}+a_{-1} z^{-1}$ and suppose $\lambda_{0}$ is a simple point. This means that the zeros of $a(z)-\lambda_{0}$ are $z_{0}, e^{i \varphi_{0}} z_{0}, w_{0}$ with $\varphi_{0} \in(-\pi, \pi] \backslash\{0\}$ and $\left|z_{0}\right|<\left|w_{0}\right|$. We have

$$
a(z)-\lambda_{0}=a_{2}\left(z-z_{0}\right)\left(z-e^{i \varphi_{0}} z_{0}\right)\left(z-w_{0}\right) z^{-1}
$$

and thus

$$
a^{\prime}\left(z_{0}\right)-e^{i \varphi_{0}} a^{\prime}\left(e^{i \varphi_{0}} z_{0}\right)=\left(1-e^{i \varphi_{0}}\right)\left(z_{0}+e^{i \varphi_{0}} z_{0}-2 w_{0}\right) .
$$

Since $e^{i \varphi_{0}} \neq 1$ and $\left|z_{0}+e^{i \varphi_{0}} z_{0}\right| \leq 2\left|z_{0}\right|<2\left|w_{0}\right|$, the right-hand side of (3.3) cannot be zero.

The rest of the paper is devoted to tetradiagonal Toeplitz matrices. In that case we accompany the topological classification of the point in $\Lambda(a)$ into regular end exceptional points by the following analytical classification. In accordance with the definition given above, we call $\lambda \in \Lambda(a)$ a simple point if

$$
\left|z_{1}(\lambda)\right|=\left|z_{2}(\lambda)\right|<\left|z_{3}(\lambda)\right| \quad \text { and } \quad z_{1}(\lambda) \neq z_{2}(\lambda) .
$$

We refer to a point $\lambda \in \Lambda(a)$ as a branch point if two (or all three) of the points $z_{1}(\lambda), z_{2}(\lambda), z_{3}(\lambda)$ coincide. In that case we may label the points so that

$$
z_{1}(\lambda)=z_{2}(\lambda) \quad \text { and } \quad\left|z_{1}(\lambda)\right|=\left|z_{2}(\lambda)\right| \leq\left|z_{3}(\lambda)\right|
$$

The remaining points $\lambda \in \Lambda(a)$ are the points for which $z_{1}(\lambda), z_{2}(\lambda), z_{3}(\lambda)$ are three distinct points satisfying

$$
\left|z_{1}(\lambda)\right|=\left|z_{2}(\lambda)\right|=\left|z_{3}(\lambda)\right| .
$$

These points will be called multiple points.

\section{Branch Points}

Let for the moment $a(z)=\sum_{j=-r}^{s} a_{j} z^{j}$ with $r, s \geq 1$ and $a_{-r} a_{s} \neq 0$ be a general Laurent polynomial. A point $\lambda_{0} \in \Lambda(a)$ is called a branch point if the equation $\lambda_{0}=a(z)$ has a root of multiplicity at least 2 . This is equivalent to saying that $\lambda_{0}=a\left(z_{0}\right)$ and that $z_{0}$ is a multiple zero of the polynomial $z^{r}\left(a(z)-\lambda_{0}\right)$. The latter happens if and only if $z_{0}$ is a root of

$$
\frac{d}{d z}\left(z^{r}\left(a(z)-\lambda_{0}\right)\right)=r z^{r-1}\left(a(z)-\lambda_{0}\right)+z^{r} a^{\prime}(z)
$$

and since $z_{0}=0$ is impossible, we conclude that $\lambda_{0} \in \Lambda(a)$ is a branch point if and only if there is a $z_{0}$ such that $\lambda=a\left(z_{0}\right)$ and $a^{\prime}\left(z_{0}\right)=0$. As

$$
a^{\prime}(z)=\sum_{j=-r}^{s} j a_{j} z^{j-1}=z^{-r-1}\left(-r a_{-r}+\cdots+s a_{s} z^{r+s}\right)
$$

the number of branch points is less than or equal to $r+s$. (The argument employed in [2, Lemma 11.4] gave only the upper bound $2(r+s)-1$.) 


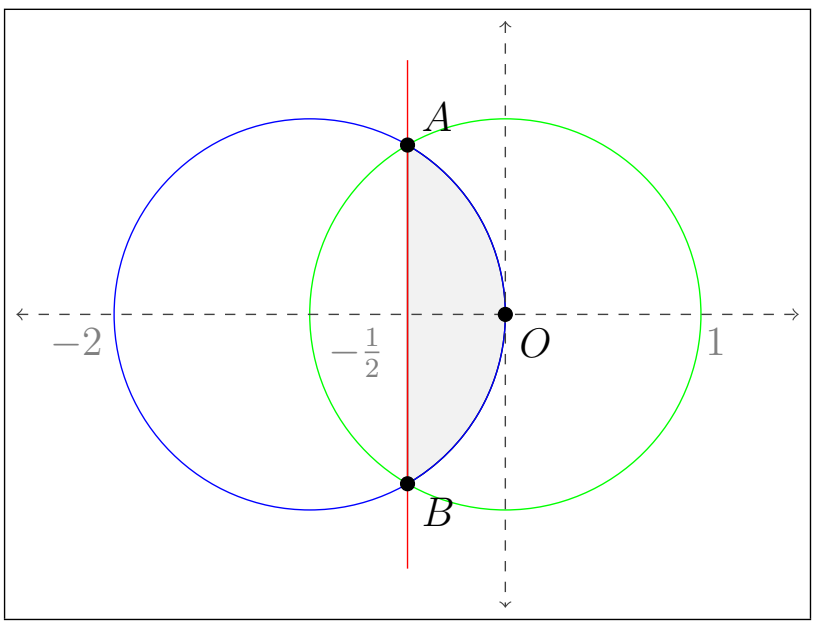

Figure 6 . The gray set $O A B$ is $\{b \in \mathbb{C}: \operatorname{Re} b \geq-1 / 2, \mid 1+$ $b \mid \leq 1\}$

We now return to the tetradiagonal case $a(z)=z^{2}+c z+c z^{-1}$ with $c \neq 0$. Then

$$
a^{\prime}(z)=\frac{2 z^{3}+c z^{2}-c}{z^{2}}
$$

has three (possibly coinciding) zeros. We denote these zeros by $t_{1}, t_{2}, t_{3}$ and label them so that $\left|t_{1}\right| \geq\left|t_{2}\right| \geq\left|t_{3}\right|$.

Lemma 4.1. There is a complex number $b \neq 0$ satisfying $\operatorname{Re} b \geq-1 / 2$ and $|1+b| \leq 1$ such that

$$
t_{2}=-\frac{b}{1+b} t_{1} \quad \text { and } \quad t_{3}=b t_{1},
$$

and after fixing any value of $w=\left(1+b+b^{2}\right)^{1 / 2}$ there is an $\sigma \in\{-1,1\}$ such that

$$
t_{1}=\sigma \frac{w}{b}, \quad t_{2}=-\sigma \frac{w}{1+b}, \quad t_{3}=\sigma w, \quad c=-2 \sigma \frac{w^{3}}{b(1+b)} .
$$

Proof. Vieta's theorem for the equation $2 z^{3}+c z^{2}-c=0$ gives

$$
t_{1} t_{2} t_{3}=\frac{c}{2}, \quad t_{1} t_{2}+t_{1} t_{3}+t_{2} t_{3}=0, \quad t_{1}+t_{2}+t_{3}=-\frac{c}{2} .
$$

Put $b^{\prime}=t_{2} / t_{1}$ and $b=t_{3} / t_{1}$. Then $0<|b| \leq\left|b^{\prime}\right| \leq 1$. The second equality of (4.3) implies that $t_{1}^{2}\left(b^{\prime}+b+b^{\prime} b\right)=0$ with $t_{1} \neq 0$. It follows that $b \neq-1$ and $b^{\prime}=-b /(1+b)$. From $0<|b| \leq\left|b^{\prime}\right| \leq 1$ we therefore get $0<|b| \leq|1+b| \leq 1$. With $b=\alpha+i \beta$, this is equivalent to the requirement $0<\alpha^{2}+\beta^{2} \leq(1+$ $\alpha)^{2}+\beta^{2} \leq 1$, which happens if and only if $b \neq 0, \alpha \geq-1 / 2,(1+\alpha)^{2}+\beta^{2} \leq 1$.

Inserting (4.1) in the first and third equalities of (4.3) we obtain

$$
-t_{1}^{3} \frac{b^{2}}{1+b}=\frac{c}{2}, \quad t_{1} \frac{1+b+b^{2}}{1+b}=-\frac{c}{2},
$$




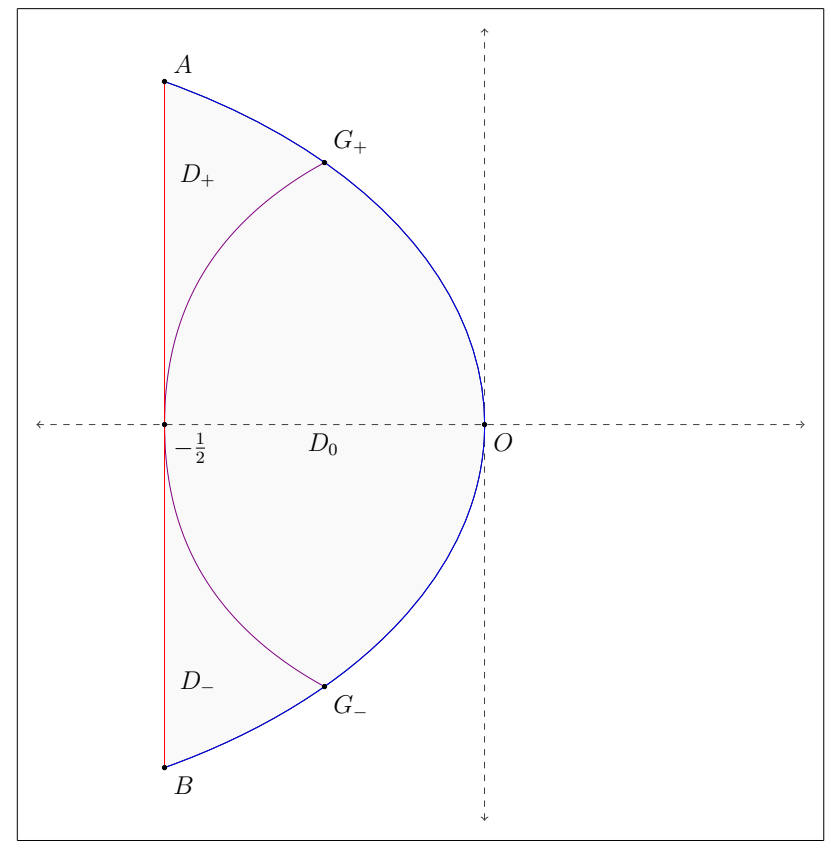

Figure 7. The set $O B A$ and the curve $\gamma$ (violet)

and hence $t_{1}^{2} b^{2}=1+b+b^{2}$. Letting $w$ be any square root of $1+b+b^{2}$, we conclude that $t_{1}=\sigma w / b$. Equalities (4.1) then give $t_{2}=-\sigma w /(1+b)$, $t_{3}=\sigma w$, and finally we have $c=2 t_{1} t_{2} t_{3}=-2 \sigma w^{3} /(b(1+b))$.

Let $\gamma$ be the piece of the curve $\left\{b \in \mathbb{C}:|1+b|=2|b|^{2}\right\}$ that lies in the open disk $\{b \in \mathbb{C}:|1+b|<1\}$. In polar coordinates,

$$
\gamma=\left\{r e^{i \varphi}: \cos \varphi=\frac{4 r^{4}-r^{2}-1}{2 r}, r \in\left(\frac{1}{2}, \frac{\sqrt{2}}{2}\right)\right\} .
$$

The endpoints of $\gamma$ on the circle $|1+b|=1$ are $G_{ \pm}=-1 / 4 \pm i \sqrt{7} / 4$. The curve $\gamma$ divides the domain $\{b \in \mathbb{C}: \operatorname{Re} b>-1 / 2,|1+b|<1\}$ into three open sets $D_{0}, D_{+}, D_{-}$as seen in Fig. 7 .

Lemma 4.2. For $k=1,2,3$, let $t_{k}$ and $b$ be as in Lemma 4.1 and put $\lambda_{k}=$ $a\left(t_{k}\right)$. Then $\lambda_{2}$ and $\lambda_{3}$ are branch points belonging to $\Lambda(a)$. The point $\lambda_{1}$ is a branch point lying on $\Lambda(a)$ if and only if $b$ is in the closure of $D_{-} \cup D_{+}$.

Proof. Let $t_{k}, t_{k}, z_{k}$ be the roots of the equation

$$
a(z)-\lambda_{k}=z^{-1}\left(z^{3}+c z^{2}-\lambda_{k} z+c\right)=0 .
$$

We have $\lambda_{k} \in \Lambda(a)$ if and only if $\left|t_{k}\right| \leq\left|z_{k}\right|$. Vieta tells us that $t_{k}^{2} z_{k}=-c$ and from (4.3) we know that $c=2 t_{1} t_{2} t_{3}$. Thus, $\left|z_{k}\right|=2\left|t_{1} t_{2} t_{3} / t_{k}^{2}\right|$. It follows that $\left|t_{k}\right| \leq\left|z_{k}\right|$ if and only if $\left|t_{k}\right|^{3} \leq 2\left|t_{1} t_{2} t_{3}\right|$. 
If $k=3$, Lemma 4.1 shows that $\left|t_{3}\right|^{3} \leq 2\left|t_{1} t_{2} t_{3}\right|$ if and only if $1 \leq$ $2 /(|b||1+b|)$. But the latter inequality is certainly true because $|b| \leq 1$ and $|1+b| \leq 1$. For $k=2$, the inequality $\left|t_{2}\right|^{3} \leq 2\left|t_{1} t_{2} t_{3}\right|$ reads $|b| \leq 2|1+b|^{2}$ due to Lemma 4.1, and this is also always true since $1 / 2 \leq|1+b|$ and $|b| \leq|1+b|$, which gives $2|1+b|^{2} \geq|1+b| \geq|b|$. Finally, in the case $k=1$, we obtain from Lemma 4.1 that $\left|t_{1}\right|^{3} \leq 2\left|t_{1} t_{2} t_{3}\right|$ if and only if $|1+b| \leq 2|b|^{2}$, which is equivalent to the requirement that $b$ is in the closure of $D_{-} \cup D_{+}$.

The blue set $\Omega$ in Fig. 2 is closed. Its left and right points are $\pm 3 \sqrt{3}$, and the upper and lower points are $\pm i$. The set $\Omega$ is symmetric about the real and the imaginary axes. Thus, it results from one of its quarters lying in one of the four quadrants by reflexions on the real and imaginary axes. Recall the connection between $b$ and $c$ given by Lemma 4.1. In dependence of the choice of $\sigma w^{3}$, the closure of $D_{+}$is mapped by

$$
b \longmapsto-2 \sigma \frac{w^{3}}{b(1+b)}=c
$$

onto either the quarter in the first quadrant or the quarter in the third quadrant. To be specific, suppose this quarter is the one in the first quadrant. Then the segment from $b=-1 / 2$ to $A$ is mapped to the segment from the (red) point $3 \sqrt{3}$ to the origin, the arc from $A$ to $G_{+}$is mapped to the segment from 0 to $i$, and the arc of $\gamma$ from $G_{+}$to $b=-1 / 2$ is mapped to the boundary piece of $\Omega$ from $i$ to $3 \sqrt{3}$. The other choice $-\sigma w^{3}$ maps the closure of $D_{+}$ to the quarter of $\Omega$ in the third quadrant. The closure of $D_{-}$is mapped into the union of the quarters in the second and fourth quadrants.

Combining the description of $\Omega$ with Lemma 4.1 we arrive at the following result.

Theorem 4.3. Let $a(z)=z^{2}+c z+c z^{-1}$ with $c \neq 0$. Denote the roots of $a^{\prime}(z)=0$ by $t_{1}, t_{2}, t_{3}$ and order them so that $\left|t_{3}\right| \leq\left|t_{2}\right| \leq\left|t_{1}\right|$. Put $\lambda_{k}=a\left(t_{k}\right)$. Then $\lambda_{2}$ and $\lambda_{3}$ are branch points of $\Lambda(a)$, whereas $\lambda_{1}$ is a branch point of $\Lambda(a)$ if and only if $c \in \Omega$.

Remark 4.4. The parameter $c$ is immediately available but checking whether $c$ is in $\Omega$ or on $\partial \Omega$ may be difficult if $c$ is close to $\partial \Omega$. However, one may proceed as follows. First solve the cubic equation $a^{\prime}(z)=0$, denote the roots by $t_{1}, t_{2}, t_{3}$, label them so that $\left|t_{1}\right| \geq\left|t_{2}\right| \geq\left|t_{3}\right|$, and put $b=t_{3} / t_{1}$. Then

$$
c \in \operatorname{int} \Omega \Longleftrightarrow|1+b|<2|b|^{2}, \quad c \in \partial \Omega \Longleftrightarrow|1+b|=2|b|^{2} .
$$

We now turn to the following question: how many analytic arcs do come out of the branch points and what is their asymptotic behavior near the branch points? Here is the result.

Theorem 4.5. Let $a(z)=z^{2}+c z+c z^{-1}$ with $c \neq 0$. Denote the roots of $a^{\prime}(z)=0$ by $t_{1}, t_{2}, t_{3}$ and order them so that $\left|t_{3}\right| \leq\left|t_{2}\right| \leq\left|t_{1}\right|$. Put $\lambda_{k}=a\left(t_{k}\right)$.

(a) If $c \notin \Omega$, then there are two analytic arcs $\ell_{2}$ and $\ell_{3}$ belonging to $\Lambda(a)$ and coming from the points $\lambda_{2}=a\left(t_{2}\right)$ and $\lambda_{3}=a\left(t_{3}\right)$, respectively, and 
these arcs have the following asymptotic representations in a neighborhood of the points $\lambda_{2}, \lambda_{3}$ :

$$
\ell_{j}=\left\{\lambda \in \mathbb{C}: \lambda=\lambda_{j}+\frac{a^{\prime \prime}\left(t_{j}\right)}{2} d_{1, j}^{2} \varphi^{2}+O\left(\varphi^{3}\right), \varphi \in(0, \varepsilon)\right\}
$$

where $d_{1, j}=-i \frac{t_{j}}{2} \quad(j=2,3)$.

(b) If $c \in$ int $\Omega$, then there are three analytic arcs $\ell_{1}, \ell_{2}$, and $\ell_{3}$ belonging to $\Lambda(a)$ and coming from the points $\lambda_{1}=a\left(t_{1}\right), \lambda_{2}=a\left(t_{2}\right)$ and $\lambda_{3}=a\left(t_{3}\right)$, respectively, and these arcs have an asymptotic representation of the form (4.4) for $j=1,2,3$.

(c) If $c \in \partial \Omega \backslash\{ \pm 3 \sqrt{3}\}$, then there are two analytic arcs that come from $\lambda_{2}=a\left(t_{2}\right)$ and $\lambda_{3}=a\left(t_{3}\right)$ with asymptotic representations of the form (4.4) in a neighborhood of these points. The roots of the equation $a(z)=$ $\lambda_{1}$ are $t_{1}, t_{1}, e^{i \varphi_{0}} t_{1}$ and there are two analytic arcs $\ell_{1}^{ \pm}$that come from $\lambda_{1}=a\left(t_{1}\right)$ and have the representations

$$
\begin{aligned}
\ell_{1}^{ \pm} & =\left\{\lambda \in \mathbb{C}: \lambda=a\left(t_{1}\right)+\frac{a^{\prime \prime}\left(t_{1}\right)}{2} d_{1}^{2}\left(\varphi-\varphi_{0}\right)^{2}+\right. \\
& \left.+\left(a^{\prime \prime}\left(t_{1}\right) d_{1} d_{2}+\frac{a^{\prime \prime \prime}\left(t_{1}\right)}{6} d_{1}^{3}\right)\left(\varphi-\varphi_{0}\right)^{3}+O\left(\left|\varphi-\varphi_{0}\right|^{4}\right)\right\},
\end{aligned}
$$

with

$$
d_{1}=-i t_{1}, \quad d_{2}=-\frac{1}{2}\left(2+i \cot \frac{\varphi_{0}}{2}\right) t_{1},
$$

where $\varphi \in\left(\varphi_{0}, \varphi_{0}+\varepsilon\right)$ for $\ell_{1}^{+}$and $\varphi \in\left(\varphi_{0}-\varepsilon, \varphi_{0}\right)$ for $\ell_{1}^{-}$. These two arcs make a cusp at the point $\lambda_{1}$.

(d) If $c= \pm \mathbf{3} \sqrt{3}$, then $\Lambda(a)$ is the line segment $\left[-9, \frac{45}{4}\right]=[-9,11.25]$.

Proof. Let $\lambda_{0} \in \Lambda(a)$ be a branch point and let $t_{0} \in\left\{t_{1}, t_{2}, t_{3}\right\}$ be a point such that $a\left(t_{0}\right)=\lambda_{0}$ and $a^{\prime}\left(t_{0}\right)=0$. The roots of the equation $a(z)=\lambda_{0}$ are $t_{0}, t_{0}, z_{3}$. From the proof of Lemma 4.2 we know that $\left|t_{0}\right|<\left|z_{3}\right|$ if and only if $\left|t_{0}\right|^{3}<2\left|t_{1} t_{2} t_{3}\right|$. The latter inequality is always true for $t_{0} \in\left\{t_{2}, t_{3}\right\}$ and it is satisfied for $t_{0}=t_{1}$ if and only if $|1+b|<2|b|^{2}$, which happens if and only if $c \in \operatorname{int} \Omega$. We know from Sect. 2 that if $\lambda \in \Lambda(a)$ is sufficiently close to $\lambda_{0}$, then $\lambda=a(u(\varphi))$ with a function $u=u(\varphi)$ that is implicitly given by the equation $a(u)-a\left(e^{i \varphi} u\right)=0$ and $u(0)=t_{0}$. The solutions of the equation $a(z)=\lambda$ are $u(\varphi), e^{i \varphi} u(\varphi), v(\varphi)$ with $u(\varphi)$ close to $t_{0}$ and $v(\varphi)$ close to $z_{3}$. Thus, if $\left|t_{0}\right|<\left|z_{3}\right|$, then any $u(\varphi)$ satisfying $a(u)-a\left(e^{i \varphi} u\right)=0$ and $u(0)=t_{0}$ yields an arc starting at $t_{0}$. We may suppose that $\varphi$ ranges over $(0, \varepsilon)$ with sufficiently small $\varepsilon>0$, because, as noted in Sect. $2, a(u(-\varphi))=a(u(\varphi))$.

We look for $u(\varphi)$ in the form

$$
u(\varphi)=t_{0}+d_{1} \varphi+d_{2} \varphi^{2}+O\left(\varphi^{3}\right) .
$$

Then

$$
\begin{aligned}
e^{i \varphi} u(\varphi) & =\left(1+i \varphi-\frac{\varphi^{2}}{2}+O\left(\varphi^{3}\right)\right)\left(t_{0}+d_{1} \varphi+d_{2} \varphi^{2}+O\left(\varphi^{3}\right)\right) \\
& =t_{0}+\left(d_{1}+i t_{0}\right) \varphi+\left(d_{2}+i d_{1}-\frac{t_{0}}{2}\right) \varphi^{2}+O\left(\varphi^{3}\right) .
\end{aligned}
$$


Taking into account that

$$
a(z)=a\left(t_{0}\right)+\frac{a^{\prime \prime}\left(t_{0}\right)}{2}\left(z-t_{0}\right)^{2}+\frac{a^{\prime \prime \prime}\left(t_{0}\right)}{6}\left(z-t_{0}\right)^{3}+O\left(\left|z-t_{0}\right|^{4}\right)
$$

we get $a(u)-a\left(e^{i \varphi} u\right)=B_{1} \varphi^{2}+B_{2} \varphi^{3}+O\left(\varphi^{4}\right)$ with

$$
\begin{aligned}
B_{1}= & \frac{a^{\prime \prime}\left(t_{0}\right)}{2}\left(d_{1}^{2}-\left(d_{1}+i t_{0}\right)^{2}\right) \\
B_{2}= & \frac{a^{\prime \prime}\left(t_{0}\right)}{2}\left(2 d_{1} d_{2}-2\left(d_{1}+i t_{0}\right)\left(d_{2}+i d_{1}-\frac{t_{0}}{2}\right)\right) \\
& +\frac{a^{\prime \prime \prime}\left(t_{0}\right)}{6}\left(d_{1}^{3}-\left(d_{1}+i t_{0}\right)^{3}\right) .
\end{aligned}
$$

The coefficients $B_{1}$ and $B_{2}$ must be zero. If $a^{\prime \prime}\left(t_{0}\right)=0$, then $c=-t_{0}^{3}$, and as also $a^{\prime}\left(t_{0}\right)=0$, we conclude that $t_{0}= \pm \sqrt{3}$ and hence $c= \pm 3 \sqrt{3}$. This case will be treated separately in part $(\mathrm{d})$. Thus, let $a^{\prime \prime}\left(t_{0}\right) \neq 0$. Then the equation $B_{1}=0$ yields

$$
d_{1}=-i \frac{t_{0}}{2}
$$

It follows that $B_{2}$ equals

$$
\begin{aligned}
& \frac{a^{\prime \prime}\left(t_{0}\right)}{2}\left[-i t_{0} d_{2}-2\left(-\frac{i t_{0}}{2}+i t_{0}\right)\left(d_{2}+\frac{t_{0}}{2}-\frac{t_{0}}{2}\right)\right] \\
& +\frac{a^{\prime \prime \prime}\left(t_{0}\right)}{6}\left[\frac{i t_{0}^{3}}{8}-\left(-\frac{i t_{0}}{2}+i t_{0}\right)^{3}\right],
\end{aligned}
$$

and hence from the equation $B_{2}=0$ we obtain

$$
-a^{\prime \prime}\left(t_{0}\right) i t_{0} d_{2}+\frac{a^{\prime \prime \prime}\left(t_{0}\right)}{6} \frac{i t_{0}^{3}}{4}=0
$$

and thus

$$
d_{2}=\frac{1}{24} \frac{a^{\prime \prime \prime}\left(t_{0}\right)}{a^{\prime \prime}\left(t_{0}\right)} t_{0}^{2} .
$$

Replacing (4.7) by $u(\varphi)=t_{0}+\sum_{k=1}^{\infty} d_{k} \varphi^{k}$, taking the full power series for $e^{i \varphi}$ and $a(z)$, and proceeding as above, one will see that for $k \geq 2$ the coefficient $d_{k}$ enters $B_{k}$ linearly and is completely determined by $d_{j}$ for $1 \leq j \leq k-1$. Consequently, there is a unique arc starting at $\lambda_{0}$. Inserting (4.7), (4.9), (4.10) into the expansion

$$
a(u)=a\left(t_{0}\right)+\frac{a^{\prime \prime}\left(t_{0}\right)}{2}\left(u-t_{0}\right)^{2}+O\left(\left|u-t_{0}\right|^{3}\right)
$$

we get the expression on the right of (4.4) for the arc. At this point we have proved all assertions concerning $\lambda_{2}$ and $\lambda_{3}$ (because for them $\left|t_{0}\right|<\left|z_{3}\right|$ ) and we have also proved part (b), since, as said, for $c \in \operatorname{int} \Omega$ we have $\left|t_{0}\right|<\left|z_{3}\right|$, too.

We turn to part (c). In that case there is a positive number $\varphi_{0}$ such that $\left\{t_{0}, t_{0}, e^{i \varphi_{0}} t_{0}\right\}$ is the solution set of the equation $a(z)=\lambda_{0}$. We have to look for solutions $u=u(\varphi)$ of the equation $a(u)-a\left(e^{i \varphi} u\right)=0$ satisfying $u(0)=t_{0}$ or $u\left(\varphi_{0}\right)=e^{i \varphi_{0}} t_{0}$. As noted above, for the initial condition $u(0)=t_{0}$ we 
need only take $\varphi$ from $[0, \varepsilon)$, but the initial condition $u\left(\varphi_{0}\right)=e^{i \varphi_{0}} t_{0}$ requires taking $\varphi$ from $\left(\varphi_{0}-\varepsilon, \varphi_{0}+\varepsilon\right)$.

We first consider the initial condition $u(0)=t_{0}$. As above, we get a unique solution $u(\varphi)=t_{0}+\sum_{k=1}^{\infty} d_{k} \varphi^{k}$ with $d_{1}, d_{2}$ given by (4.9), (4.10). Vieta for the equation

$$
z\left(a(z)-\lambda_{0}\right)=z^{3}+c z^{2}-\lambda_{0} z+c=0
$$

gives $2 t_{0}+e^{i \varphi_{0}} t_{0}=-c$ and $e^{i \varphi_{0}} t_{0}^{3}=-c$. This implies

$$
a^{\prime \prime}\left(t_{0}\right)=2\left(1-e^{i \varphi_{0}}\right), \quad a^{\prime \prime \prime}\left(t_{0}\right)=6 \frac{e^{i \varphi_{0}}}{t_{0}}
$$

and results in

$$
d_{2}=\frac{e^{i \varphi_{0}} t_{0}}{8\left(1-e^{i \varphi_{0}}\right)}=\frac{1}{16} i \frac{e^{i \varphi_{0} / 2} t_{0}}{\sin \left(\varphi_{0} / 2\right)} .
$$

Consequently,

$$
\begin{aligned}
|u(\varphi)|^{2} & =\left|t_{0}\right|^{2}\left|1-\frac{i}{2} \varphi+\frac{i}{16} \frac{e^{i \varphi_{0} / 2}}{\sin \left(\varphi_{0} / 2\right)} \varphi^{2}+O\left(\varphi^{3}\right)\right|^{2} \\
& =\left|t_{0}\right|^{2}\left(\left(1-\frac{1}{16} \varphi^{2}\right)^{2}+\left(-\frac{1}{2} \varphi\right)^{2}+O\left(\varphi^{3}\right)\right) \\
& =\left|t_{0}\right|^{2}\left(1+\frac{1}{8} \varphi^{2}+O\left(\varphi^{3}\right)\right) .
\end{aligned}
$$

It follows that $|u(\varphi)|>\left|t_{0}\right|$. The zeros of $z(a(z)-\lambda)=z^{3}+c z^{2}-\lambda z+c$ are $u(\varphi), e^{i \varphi} u(\varphi), v(\varphi)$, and Vieta tells us that $e^{i \varphi} u^{2}(\varphi) v(\varphi)=-c$. Thus,

$$
|v(\varphi)|=\frac{|c|}{|u(\varphi)|^{2}}<\frac{|c|}{\left|t_{0}\right|^{2}}=|v(0)|=\left|t_{0}\right|<|u(\varphi)|,
$$

and because $\lambda \in \Lambda(a)$ would require that $|u(\varphi)|=\left|e^{i \varphi} u(\varphi)\right| \leq|v(\varphi)|$ we conclude that (4.7) does not give an arc of $\Lambda(a)$.

We are left with the equation $a(u)-a\left(e^{i \varphi} u\right)=0$ in neighborhood of the point $\varphi=\varphi_{0}$. Using power series one can show that the solution is unique. We may write

$u(\varphi)=t_{0}+d_{1}\left(\varphi-\varphi_{0}\right)+d_{2}\left(\varphi-\varphi_{0}\right)^{2}+O\left(\left|\varphi-\varphi_{0}\right|^{3}\right), \quad \varphi \in\left(\varphi_{0}-\varepsilon, \varphi_{0}+\varepsilon\right)$

and calculations as before give

$$
d_{1}=-i t_{0}, \quad d_{2}=-\frac{1}{2}\left(2+i \cot \frac{\varphi_{0}}{2}\right) t_{0} .
$$

It follows that

$$
\begin{aligned}
|u(\varphi)|^{2}= & \left|t_{0}\right|^{2}\left(1-i\left(\varphi-\varphi_{0}\right)-\frac{1}{2}\left(2+i \cot \frac{\varphi_{0}}{2}\right)\left(\varphi-\varphi_{0}\right)^{2}\right. \\
& \left.+O\left(\left|\varphi-\varphi_{0}\right|^{3}\right)\right)^{2} \\
= & \left|t_{0}\right|^{2}\left(\left(1-\left(\varphi-\varphi_{0}\right)^{2}\right)^{2}+\left(\left(-\left(\varphi-\varphi_{0}\right)\right)^{2}+O\left(\left|\varphi-\varphi_{0}\right|^{3}\right)\right)\right. \\
= & \left|t_{0}\right|^{2}\left(1-\left(\varphi-\varphi_{0}\right)^{2}+O\left(\left|\varphi-\varphi_{0}\right|^{3}\right)\right) .
\end{aligned}
$$


This time $|u(\varphi)|<\left|t_{0}\right|$ and hence

$$
|v(\varphi)|=\frac{|c|}{|u(\varphi)|^{2}}>\frac{|c|}{\left|t_{0}\right|^{2}}=\frac{|c|}{\left|u\left(\varphi_{0}\right)\right|^{2}}=\left|v\left(\varphi_{0}\right)\right|=\left|t_{0}\right|>|u(\varphi)|,
$$

which shows that the two arcs induced by $\varphi \in\left(\varphi_{0}-\varepsilon, \varphi_{0}\right)$ and $\varphi \in\left(\varphi_{0}, \varphi_{0}+\varepsilon\right)$ are part of $\Lambda(a)$. Inserting the expressions obtained for $u(\varphi)$ in (4.11) with $t_{0}$ replaced by $t_{1}$ we get (4.5) and (4.6). It is clear that $\ell_{1}^{ \pm}$make a cusp at $\lambda_{1}$.

We finally prove (d). Equality (1.3) with $\varrho=-1$ shows that always

$$
\Lambda\left(z^{2}+c z+c z^{-1}\right)=\Lambda\left(z^{2}-c z-c z^{-1}\right)
$$

Thus, the limiting sets for $c= \pm 3 \sqrt{3}$ are the same. Let $a(z)=z^{2}-c z-c z^{-1}$ with $c=3 \sqrt{3}$. Using (1.3) with $\varrho=\sqrt{3}$, we get

$$
\Lambda(a)=\Lambda\left(a_{\sqrt{3}}\right)=\Lambda\left(3 z^{2}-3 \sqrt{3} \sqrt{3} z-\frac{3 \sqrt{3}}{\sqrt{3}} z^{-1}\right)=3 \Lambda\left(z^{2}-3 z-z^{-1}\right) .
$$

Consequently, with $f(z):=z^{2}-3 z-z^{-1}$, we have $\Lambda(a)=3 \Lambda(f)$ and we are left with proving that $\Lambda(f)=[-3,15 / 4]$.

We first show that $\Lambda(f)$ is a subset of $\mathbb{R}$. So let $\lambda \in \Lambda(f)$. From Sect. 2 we know that $\lambda=f(z)$ where $z$ satisfies $f(z)-f\left(e^{i \varphi} z\right)=0$ for some $\varphi \neq 0$ or where $z=t_{k}$ is a root of the equation $f^{\prime}(z)=0$. The zeros of $f^{\prime}(z)$ are $t_{1}=1, t_{2}=1, t_{3}=-1 / 2$ and $\lambda_{2}=f\left(t_{2}\right)=-3$ as well as $\lambda_{3}=f\left(t_{3}\right)=15 / 4$ are real. So suppose $f(z)-f\left(e^{i \varphi} z\right)=0$ with $\varphi \neq 0$. Adding 3 to both sides of the equation $f(z)=f\left(e^{i \varphi} z\right)$ we obtain the equation

$$
\frac{(z-1)^{3}}{z}=\frac{\left(e^{i \varphi} z-1\right)^{3}}{e^{i \varphi} z}
$$

and writing $v:=e^{i \varphi / 3}$, this equation becomes

$$
\left(\frac{v^{2} z-v^{-1}}{z-1}\right)^{3}=1
$$

Consequently, $\left(v^{2} z-v^{-1}\right) /(z-1)=\varepsilon$ where $\varepsilon^{3}=1$. It follows at once that $z=\left(v^{-1}-\varepsilon\right) /\left(v^{2}-\varepsilon\right)$ and thus

$$
\lambda=f(z)=\frac{(z-1)^{3}}{z}-3=\frac{\left(1-v^{3}\right)^{3}}{v^{2}\left(v^{2}-\varepsilon\right)^{2}(1-v \varepsilon)}-3 .
$$

This gives

$$
\begin{aligned}
\bar{\lambda} & =\frac{\left(1-v^{-3}\right)^{3}}{v^{-2}\left(v^{-2}-\varepsilon^{-1}\right)^{2}\left(1-v^{-1} \varepsilon^{-1}\right)}-3 \\
& =\frac{v^{-9}\left(v^{3}-1\right)^{3}}{\left(v^{-2} v^{-1} v^{-4}\right)\left(\varepsilon^{-1} \varepsilon^{-2}\right)\left(\varepsilon-v^{2}\right)(v \varepsilon-1)}-3=\lambda
\end{aligned}
$$

and therefore implies that $\lambda \in \mathbb{R}$, as asserted.

In summary, $\Lambda(f)$ is a closed and connected subset of $\mathbb{R}$ and thus some closed line segment in $\mathbb{R}$. To conclude that $\Lambda(f)=[-3,15 / 4]$, we may have recourse to part (a), according to which $\Lambda(f)$ is an analytic arc starting at $\lambda_{2}=f\left(t_{2}\right)=-3$ and terminating at $\lambda_{3}=f\left(t_{3}\right)=15 / 4$. An argument avoiding part (a) is as follows. We know that $\Lambda(f)$ is the intersection of the spectra of the Toeplitz operators $T\left(f_{\varrho}\right)$ for $\varrho \in(0, \infty)$. So consider the curve 
$f_{\varrho}(\mathbb{T})$. If $\varrho=1$, this curve and thus also the spectrum of $T\left(f_{\varrho}\right)$ are located in the half-plane $\operatorname{Re} \lambda \geq-3$, and if $\varrho=-1 / 2$, the curve and hence the spectrum of $T\left(f_{\varrho}\right)$ are contained in the half-plane $\operatorname{Re} \lambda \leq 15 / 4$. Consequently, $\Lambda(f)$ is a subset of $[-3,15 / 4]$, and since $-3=\lambda_{2}$ and $15 / 4=\lambda_{3}$ belong to $\Lambda(f)$, it follows that $\Lambda(f)=[-3,15 / 4]$.

Remark 4.6. The referee kindly pointed out that part of assertion (d), namely that $\Lambda(f) \subset \mathbb{R}$, may also be derived from the main result of [11], according to which $\Lambda(f)$ is contained in $\mathbb{R}$ if and only if the pre-image $f^{-1}(\mathbb{R})$ contains a Jordan curve. To see this, let $q(t)=\sin (3 t) / \sin (2 t)$ and consider the set $K=\left\{\kappa(t):=1-q e^{i t}: t \in[-\pi / 3, \pi / 3]\right\}$. This is a Jordan curve and we have $f(\kappa)=\kappa^{2}-3 \kappa-\frac{1}{\kappa}=\frac{(\kappa-1)^{3}}{\kappa}-3=-q^{3} \frac{e^{3 i t}}{\kappa}-3=-\frac{q^{3}}{|\kappa|^{2}} e^{3 i t}\left(1-q e^{-i t}\right)-3$.

Consequently, the imaginary part of $f(\kappa(t))$ is

$$
-\frac{q^{3}}{|\kappa|^{2}}(\sin (3 t)-q \sin (2 t))=-\frac{q^{3}}{|\kappa|^{2}}\left(\sin (3 t)-\frac{\sin (3 t)}{\sin (2 t)} \sin (2 t)\right)=0,
$$

which shows that $f(K) \subset \mathbb{R}$ and thus, by $[11], \Lambda(f) \subset \mathbb{R}$.

We recall that $t_{1}, t_{2}, t_{3}$ are the zeros of $a^{\prime}(z)$. We therefore have

$$
a^{\prime}\left(t_{k}\right)=2 t_{k}+c-\frac{c}{t_{k}^{2}}=0, \quad a^{\prime \prime}\left(t_{k}\right)=2+\frac{2 c}{t_{k}^{3}} .
$$

The first of these equalities gives $c / t_{k}^{3}=2+c / t_{k}$, and inserting this in the second equality we get $a^{\prime \prime}\left(t_{k}\right)=6+2 c / t_{k}$. Thus the repeatedly occurring $a^{\prime \prime}\left(t_{k}\right)$ in the theorem may simply be replaced by $6+2 c / t_{k}$.

\section{Multiple Points}

Let again $a(z)=z^{2}+c z+c z^{-1}$ with $c \neq 0$. Recall that a point $\lambda_{0} \in \Lambda(a)$ is said to be a multiple point if the roots $z_{1}\left(\lambda_{0}\right), z_{2}\left(\lambda_{0}\right), z_{3}\left(\lambda_{0}\right)$ of the equation $a(z)=\lambda_{0}$ are pairwise distinct but have the same absolute value.

Lemma 5.1. Let $r=|c|^{1 / 3}$ and write $c=-r^{3} e^{i \theta}$ with $\theta \in(-\pi, \pi]$. Put $d=$ $r^{2} e^{2 i \theta / 3}$. Then the following are equivalent:

(i) there is a point $\lambda_{0} \in \Lambda(a)$ such that the equation $a(z)=\lambda_{0}$ has three solutions of the same absolute value,

(ii) the equation

$$
h_{p}(\psi):=p e^{-i \psi}+e^{2 i \psi}=d
$$

has a solution $(\psi, p) \in \mathbb{R} \times[-2,2]$.

If (i), (ii) hold, then $\lambda_{0}$ necessarily equals $-r^{4}\left(=-|c|^{4 / 3}\right)$.

Proof. (i) $\Rightarrow$ (ii). Suppose the equation $a(z)=\lambda_{0}$ has three solutions of the same modulus. They are $z_{k}\left(\lambda_{0}\right)=r e^{i \varphi_{k}}$ with $r>0$ and $\varphi_{k} \in \mathbb{R}$. Vieta's theorem applied to the equation

$$
a(z)-\lambda_{0}=z^{-1}\left(z^{3}+c z^{2}-\lambda_{0} z+c\right)=0
$$


gives

$$
r^{3} e^{i\left(\varphi_{1}+\varphi_{2}+\varphi_{3}\right)}=-c, \quad r\left(e^{i \varphi_{1}}+e^{i \varphi_{2}}+e^{i \varphi_{3}}\right)=-c .
$$

It follows that $r=|c|^{1 / 3}$ and $\varphi_{1}+\varphi_{2}+\varphi_{3}=\theta+\xi$ with $\xi \in 2 \pi \mathbb{Z}$. From (5.3) we now infer that

$$
e^{i\left(\varphi_{1}+\varphi_{2}\right) / 2}\left[e^{i\left(\varphi_{1}-\varphi_{2}\right) / 2}+e^{-i\left(\varphi_{1}-\varphi_{2}\right) / 2}\right]+e^{i\left(\theta+\xi-\varphi_{1}-\varphi_{2}\right)}=-\frac{c}{r},
$$

or equivalently,

$$
e^{i\left(\varphi_{1}+\varphi_{2}\right) / 2} 2 \cos \frac{\varphi_{2}-\varphi_{1}}{2}+e^{i \theta} e^{-i\left(\varphi_{1}+\varphi_{2}\right)}=-\frac{c}{r} .
$$

Putting

$$
\psi=\frac{\theta}{3}-\frac{\varphi_{1}+\varphi_{2}}{2}, \quad p=2 \cos \frac{\varphi_{2}-\varphi_{1}}{2},
$$

we get $e^{i(\theta / 3-\psi)} p+e^{i \theta} e^{-2 i(\theta / 3-\psi)}=-c / r$, that is,

$$
e^{i \theta / 3} p e^{-i \psi}+e^{i \theta / 3} e^{2 i \psi}=-\frac{c}{r},
$$

and finally

$$
h_{p}(\psi)=p e^{-i \psi}+e^{2 i \psi}=-\frac{e^{-i \theta / 3} c}{r}=-\frac{e^{-i \theta / 3}\left(-e^{i \theta}\right) r^{3}}{r}=r^{2} e^{2 i \theta / 3}=d .
$$

(ii) $\Rightarrow($ i). Let $(\psi, p) \in \mathbb{R} \times[-2,2]$ be a solution of (5.1). Then (5.6) holds, and with arbitrary $\varphi_{1}, \varphi_{2}$ satisfying (5.5), we obtain (5.4) with $\xi=0$. After putting $\varphi_{3}=\theta-\varphi_{1}-\varphi_{2}$ we arrive at (5.3). Equation (5.2) is then satisfied by $z_{k}=r e^{i \varphi_{k}}$ when taking

$$
\lambda_{0}=-r^{2}\left(e^{i\left(\varphi_{1}+\varphi_{2}\right)}+e^{i\left(\varphi_{1}+\varphi_{3}\right)}+e^{i\left(\varphi_{2}+\varphi_{3}\right)}\right) .
$$

Now suppose (i) is true. Then the solutions of the equation $a(z)=\lambda_{0}$ are $r e^{i \varphi_{k}}$ as above and Vieta implies (5.3) and (5.7). Since $\varphi_{1}+\varphi_{2}+\varphi_{3}-\theta \in 2 \pi \mathbb{Z}$, we obtain from (5.7) that

$$
r^{2}\left(e^{i\left(\theta-\varphi_{3}\right)}+e^{i\left(\theta-\varphi_{2}\right)}+e^{i\left(\theta-\varphi_{1}\right)}\right)=-\lambda_{0}
$$

and hence

$$
-\lambda_{0}=r e^{i \theta} \overline{r\left(e^{i \varphi_{1}}+e^{i \varphi_{2}}+e^{i \varphi_{3}}\right)}=r\left(-\frac{c}{r^{3}}\right)(-\bar{c})=\frac{|c|^{2}}{r^{2}}=\frac{r^{6}}{r^{2}}=r^{4} .
$$

We emphasize that in the previous lemma we admit $\varphi_{1} \leq \varphi_{2} \leq \varphi_{3}$. The cases $\varphi_{1}=\varphi_{2}$ and $\varphi_{2}=\varphi_{3}$ occur for branch points. For multiple points we have strict inequalities.

Let $D_{2}$ be the set of all $d \in \mathbb{C}$ for which Eq. (5.1) has a solution $p \in$ $[-2,2]$ and $\psi \in \mathbb{R}$. Note that the function $h_{p}: \mathbb{R} \rightarrow \mathbb{C}$ is $2 \pi$-periodic and that $h_{-p}(\mathbb{R})=h_{p}(\mathbb{R})$. Figure 8 a shows the ranges $h_{p}(\mathbb{R})$ for several values of $p$ and suggests that $D_{2}$ is the set bounded by $h_{2}(\mathbb{R})$. This can indeed be proved, but we do not need this for our purposes. Moreover, writing $-c=r^{3} e^{i \theta}$ and $d=r^{2} e^{2 \pi i / 3}$ as in Lemma 5.1, we get

$$
(-c)^{2 / 3}=\left(r^{3} e^{i \theta}\right)^{2 / 3}=\varepsilon^{k} r^{2} e^{2 \pi i / 3}=\varepsilon^{k} d \quad\left(\varepsilon=e^{2 \pi i / 3}, \quad k=0,1,2\right) .
$$




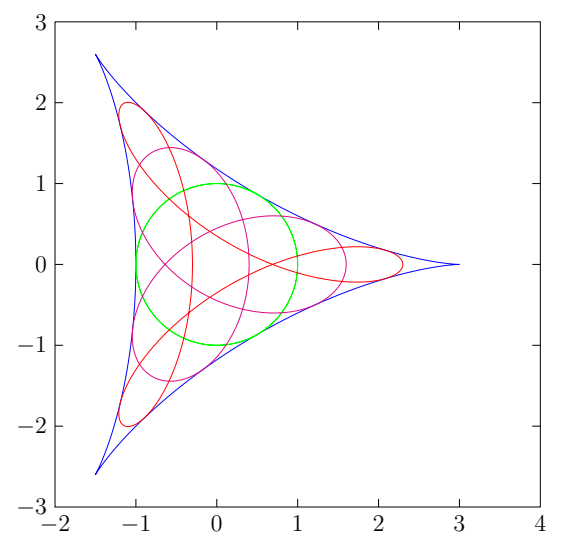

(a)

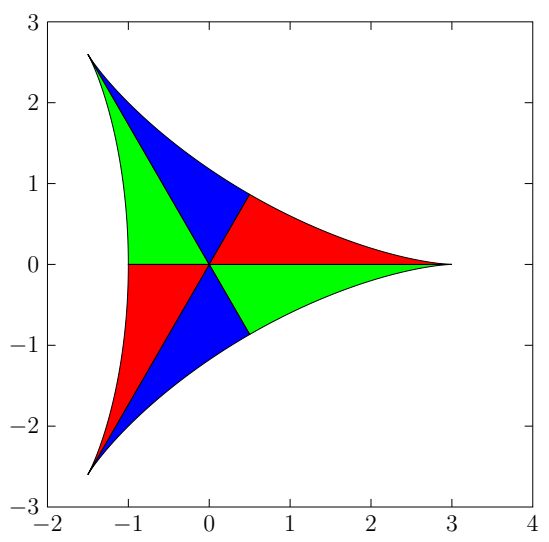

(b)

Figure 8 . On the left we see the range $h_{p}(\mathbb{R})$ for $p=0$ (green), $p=0.6$ (magenta), $p=1.3$ (red), $p=2$ (blue).On the right we show the set $\Omega^{2 / 3}$ divided into three pieces

The map $c \mapsto(-c)^{2 / 3}$ maps $\Omega$ to the set shown in Fig. 8 b. The blue set is the image of the part of $\Omega$ that lies on the right of the imaginary axis for some $k \in\{0,1,2\}$, the red and green sets are the images for the other two values of $k$. The left piece of $\Omega$ is mapped onto the same set. Thus, the set in Fig. $8 \mathrm{~b}$ is covered twice. Comparison of Fig. $8 \mathrm{a}$, b indicates that $D_{2}=(-\Omega)^{2 / 3}$, and since $\Omega=-\Omega$, that actually $D_{2}=\Omega^{2 / 3}$. This can again be rigorously verified, but we will not embark on this issue here. The conclusion is that $\Lambda(a)$ has a point $\lambda_{0}$ for which the equation $a(z)=\lambda_{0}$ has three solutions of the same modulus if and only if $(-c)^{2 / 3} \in D_{2}$ and that this happens if and only if $c \in \Omega$. The result of genuine interest to us is that $\Lambda(a)$ has a multiple point, that is, a point $\lambda_{0}$ such that $a(z)=\lambda_{0}$ is satisfied by three pairwise distinct numbers of the same modulus, if and only if $c \in$ int $\Omega$. This will be a consequence of Theorem 6.1 below.

Theorem 5.2. Let $\lambda_{0}$ be a multiple point of $\Lambda(a)$ and let $r e^{i \varphi_{1}}, r e^{i \varphi_{2}}, r e^{i \varphi_{3}}$ with

$$
-\pi<\varphi_{1}<\varphi_{2}<\varphi_{3} \leq \pi
$$

be the solutions of the equation $a(z)=\lambda_{0}$. Then there are exactly three analytic arcs of $\Lambda(a)$ coming from $\lambda_{0}$. Their local representations are

$$
\ell_{0, j}=\left\{\lambda \in \mathbb{C}: \lambda=\lambda_{0}+a^{\prime}\left(z_{0, j}\right) q_{j}\left(\varphi-\varphi_{0, j}\right)+O\left(\left|\varphi-\varphi_{0, j}\right|\right)^{2}\right\}
$$

for $j=1,2,3$ with

$$
q_{j}=\frac{i z_{0, j}}{s\left(z_{0, j}, \varphi_{0, j}\right)-1}
$$

and

$$
z_{0,1}=r e^{i \varphi_{1}}, \varphi_{0,1}=\varphi_{2}-\varphi_{1}, \quad s\left(z_{0,1}, \varphi_{0,1}\right)=-\frac{\sin \frac{\varphi_{3}-\varphi_{1}}{2}}{\sin \frac{\varphi_{3}-\varphi_{2}}{2}} e^{-\frac{i\left(\varphi_{2}-\varphi_{1}\right)}{2}},
$$




$$
\begin{aligned}
& z_{0,2}=r e^{i \varphi_{1}}, \varphi_{0,2}=\varphi_{3}-\varphi_{1}, \quad s\left(z_{0,2}, \varphi_{0,2}\right)=-\frac{\sin \frac{\varphi_{2}-\varphi_{1}}{2}}{\sin \frac{\varphi_{2}-\varphi_{3}}{2}} e^{-\frac{i\left(\varphi_{3}-\varphi_{1}\right)}{2}}, \\
& z_{0,3}=r e^{i \varphi_{2}}, \varphi_{0,3}=\varphi_{3}-\varphi_{2}, \quad s\left(z_{0,3}, \varphi_{0,3}\right)=-\frac{\sin \frac{\varphi_{1}-\varphi_{2}}{2}}{\sin \frac{\varphi_{1}-\varphi_{3}}{2}} e^{-\frac{i\left(\varphi_{3}-\varphi_{2}\right)}{2}} .
\end{aligned}
$$

In (5.8) one has to take $\varphi>\varphi_{0}$ or $\varphi<\varphi_{0}$ to enforce that

$$
\left(\operatorname{Re}\left(q_{j} / z_{0, j}\right)\right)\left(\varphi-\varphi_{0}\right)<0 .
$$

Proof. We proceed as in the case of branch points. Now the equation $a(z)-$ $\lambda_{0}=0$ has the solutions $r e^{i \varphi_{1}}, r e^{i \varphi_{2}}, r e^{i \varphi_{3}}$, where $r>0, \varphi_{j} \in(-\pi, \pi]$, and $\varphi_{1}<\varphi_{2}<\varphi_{3}$. In order to find the analytic arcs that pass through $\lambda_{0}$ in a neighborhood around it, we consider again the equation $a(u)-a\left(e^{i \varphi} u\right)=0$. We have $a\left(u_{0}\right)-a\left(e^{i \varphi_{0}} u_{0}\right)=0$ in the following three cases:

$$
\begin{array}{lll}
u_{0}=r e^{i \varphi_{1}}, & \varphi_{0}=\varphi_{2}-\varphi_{1}, & j=1, \\
u_{0}=r e^{i \varphi_{1}}, & \varphi_{0}=\varphi_{3}-\varphi_{1}, & j=2, \\
u_{0}=r e^{i \varphi_{2}}, & \varphi_{0}=\varphi_{3}-\varphi_{2}, & j=3 .
\end{array}
$$

Pick one of these cases. In a neighborhood of $\left(u_{0}, \varphi_{0}\right)$ we look for $u(\varphi)$ in the form

$$
u(\varphi)=u_{0}+q\left(\varphi-\varphi_{0}\right)+O\left(\left|\varphi-\varphi_{0}\right|^{2}\right), \quad \varphi \in\left(\varphi_{0}-\varepsilon, \varphi_{0}+\varepsilon\right)
$$

Of course, $q$ depends on $j$ and will eventually become $q_{j}$. We obtain

$$
\begin{aligned}
e^{i \varphi} u(\varphi) & =e^{i \varphi_{0}} e^{i\left(\varphi-\varphi_{0}\right)} u(\varphi)=e^{i \varphi_{0}}\left(1+i\left(\varphi-\varphi_{0}\right)+O\left(\left|\varphi-\varphi_{0}\right|^{2}\right)\right) u(\varphi) \\
& =e^{i \varphi_{0}} u_{0}+e^{i \varphi_{0}}\left(q+i u_{0}\right)\left(\varphi-\varphi_{0}\right)+O\left(\left|\varphi-\varphi_{0}\right|^{2}\right)
\end{aligned}
$$

and thus

$$
a(u)-a\left(e^{i \varphi} u\right)=\left(a^{\prime}\left(u_{0}\right) q-e^{i \varphi_{0}} a^{\prime}\left(e^{i \varphi_{0}} u_{0}\right)\left(q+i u_{0}\right)\right)\left(\varphi-\varphi_{0}\right)+O\left(\left|\varphi-\varphi_{0}\right|^{2}\right),
$$

implying that $a^{\prime}\left(u_{0}\right) q-e^{i \varphi_{0}} a^{\prime}\left(e^{i \varphi_{0}} u_{0}\right)\left(q+i u_{0}\right)=0$. This gives

$$
q=\frac{i u_{0}}{s\left(u_{0}, \varphi_{0}\right)-1} \quad \text { with } \quad s\left(u_{0}, \varphi_{0}\right):=e^{-i \varphi_{0}} \frac{a^{\prime}\left(u_{0}\right)}{a^{\prime}\left(u_{0} e^{i \varphi_{0}}\right)} .
$$

Let us calculate $a^{\prime}\left(u_{0}\right)$ at $u_{0}=r e^{i \varphi_{1}}$. We have

$$
a^{\prime}\left(r e^{i \varphi_{1}}\right)=2 r e^{i \varphi_{1}}+c-\frac{c}{r^{2} e^{2 i \varphi_{1}}} .
$$

Using the Vieta equalities (5.3) we get

$$
\begin{aligned}
a^{\prime}\left(u_{0}\right) & =a^{\prime}\left(r e^{i \varphi_{1}}\right)=r\left(2 e^{i \varphi_{1}}-\left(e^{i \varphi_{1}}+e^{i \varphi_{2}}+e^{i \varphi_{3}}\right)+e^{i\left(-\varphi_{1}+\varphi_{2}+\varphi_{3}\right)}\right) \\
& =r\left(\left(e^{i \varphi_{1}}-e^{i \varphi_{2}}\right)-e^{i \varphi_{3}}\left(1-e^{-i\left(\varphi_{1}-\varphi_{2}\right)}\right)\right) \\
& =r\left(2 i e^{i \frac{\varphi_{1}+\varphi_{2}}{2}} \sin \frac{\varphi_{1}-\varphi_{2}}{2}-2 i e^{i\left(\varphi_{3}-\frac{\varphi_{1}-\varphi_{2}}{2}\right)} \sin \frac{\varphi_{1}-\varphi_{2}}{2}\right) \\
& =2 i r \sin \frac{\varphi_{1}-\varphi_{2}}{2} e^{i \frac{\varphi_{2}}{2}}\left(e^{i \frac{\varphi_{1}}{2}}-e^{i\left(\varphi_{3}-\frac{\varphi_{1}}{2}\right)}\right) \\
& =-4 r e^{i \frac{\varphi_{2}+\varphi_{3}}{2}} \sin \frac{\varphi_{1}-\varphi_{2}}{2} \sin \frac{\varphi_{1}-\varphi_{3}}{2} .
\end{aligned}
$$


Analogously,

$$
a^{\prime}\left(e^{i \varphi_{0}} u_{0}\right)=a^{\prime}\left(r e^{i \varphi_{2}}\right)=-4 r e^{i \frac{\varphi_{1}+\varphi_{3}}{2}} \sin \frac{\varphi_{2}-\varphi_{1}}{2} \sin \frac{\varphi_{2}-\varphi_{3}}{2} .
$$

Inserting the two expressions obtained into (5.10) we arrive at

$$
s\left(u_{0}, \varphi_{0}\right)=s\left(r e^{i \varphi_{1}}, \varphi_{2}-\varphi_{1}\right)=-\frac{\sin \frac{\varphi_{3}-\varphi_{1}}{2}}{\sin \frac{\varphi_{3}-\varphi_{2}}{2}} e^{-\frac{i\left(\varphi_{2}-\varphi_{1}\right)}{2}} .
$$

We have $\operatorname{Re}\left(q / u_{0}\right) \neq 0$. Indeed, if $\operatorname{Re}\left(q / u_{0}\right)=0$, then, by $(5.10), s\left(u_{0}, \varphi_{0}\right)$ must be a real number, which implies that $\operatorname{Im}\left(e^{i\left(\varphi_{2}-\varphi_{1}\right) / 2}\right)=0$, whence $\varphi_{2}=\varphi_{1}$, contradicting our requirement $\varphi_{2}>\varphi_{1}$. From (5.9) we now get

$$
|u(\varphi)|^{2}=\left|u_{0}\right|^{2}\left(1+2 \operatorname{Re}\left(g / u_{0}\right)\left(\varphi-\varphi_{0}\right)+O\left(\left|\varphi-\varphi_{0}\right|^{2}\right) .\right.
$$

It follows that $a(u(\varphi))$ belongs to $\Lambda(a)$ if and only if

$$
\left(\operatorname{Re}\left(g / u_{0}\right)\right)\left(\varphi-\varphi_{0}\right)<0
$$

since if we have (5.11), then the roots of the equation $a(z)=\lambda(\varphi)$ with $\lambda(\varphi)=$ $a(u(\varphi))$ have absolute value smaller than $r$, which means that the third root $v(\varphi)$ of this equation has absolute value $|v(\varphi)|=\frac{|c|}{|u(\varphi)|^{2}}=\frac{r^{3}}{|u(\varphi)|^{2}}>r$, while if (5.11) does not hold, then $|v(\varphi)|<|u(\varphi)|$. The reasoning is analogous for $j=2$ and $j=3$. Thus, we have shown that three analytic arcs with the asserted local representation start at $\lambda_{0}$. Inserting the full power series for $u(\varphi)$ in the equation $a(u)-a\left(e^{i \varphi} u\right)=0$ one can check that all its coefficients are uniquely determined, which implies that no more than three analytic arcs come from $\lambda_{0}$.

\section{The Types of the Limiting Set}

We say that $\Lambda(a)$ is of type $L_{j}(j=1,2,3)$ if $\Lambda(a)$ is the closure of the union of exactly $j$ analytic arcs and $\Lambda(a)$ has exactly $j+1$ exceptional points.

Theorem 6.1. Let $a(z)=z^{2}+c z+c z^{-1}$ with $c \neq 0$. Denote the roots of $a^{\prime}(z)=0$ by $t_{1}, t_{2}, t_{3}$ and order them so that $\left|t_{3}\right| \leq\left|t_{2}\right| \leq\left|t_{1}\right|$. Put $\lambda_{k}=a\left(t_{k}\right)$.

(a) The set $\Lambda(a)$ is of type $L_{1}$ if and only if $c \notin \Omega$ or $c= \pm 3 \sqrt{3}$. In that case $\Lambda(a)=\left[\lambda_{2} \sim \lambda_{3}\right]$.

(b) The set $\Lambda(a)$ is of type $L_{2}$ if and only if $c \in \partial \Omega \backslash\{ \pm 3 \sqrt{3}\}$. In that case the set $\Lambda(a)$ is $\Lambda(a)=\left[\lambda_{1} \sim \lambda_{2}\right] \cup\left[\lambda_{1} \sim \lambda_{3}\right]$ with a cusp at $\lambda_{1}$.

(c) The set $\Lambda(a)$ is of type $L_{3}$ if and only if $c \in$ int $\Omega$. In that case we have $\Lambda(a)=\left[\lambda_{0} \sim \lambda_{1}\right] \cup\left[\lambda_{0} \sim \lambda_{2}\right] \cup\left[\lambda_{0} \sim \lambda_{3}\right]$ with $\lambda_{0}=-|c|^{4 / 3}$.

Proof. If $c= \pm 3 \sqrt{3}$, then $\Lambda(a)$ is a line segment by Theorem $4.5(\mathrm{~d})$. Let $c \notin \Omega$. Then, by Theorems 4.3 and 4.5, $\Lambda(a)$ has exactly $\lambda_{2}$ and $\lambda_{3}$ as branch points and in each of them there is one outgoing arc. If $\Lambda(a)$ had a multiple point $\lambda_{0}$, then due to Theorem 5.2 there would be three arcs starting at $\lambda_{0}$. Note that, by Lemma $5.1, \Lambda(a)$ can have at most one multiple point. It is impossible to weld these five arcs together without creating additional exceptional points and without increasing the number of outgoing arcs in 
$\lambda_{2}$ and $\lambda_{3}$. Thus, $\Lambda(a)$ cannot posses a multiple point and we must have $\Lambda(a)=\left[\lambda_{2} \sim \lambda_{3}\right]$.

Now suppose $c \in$ int $\Omega$. Then, again by Theorems 4.3 and $4.5, \lambda_{1}, \lambda_{2}, \lambda_{3}$ are branch points with a single arc starting in each of them. One cannot glue together these three arcs without creating additional exceptional points or increasing the number of outgoing arcs in the branch points. Consequently, $\Lambda(a)$ must have a multiple point $\lambda_{0}$ with three outgoing arcs. The only possibility to join these arcs in order to get a connected set with no more exceptional points and without increasing the number of outgoing arcs in the branch points is $\Lambda(a)=\left[\lambda_{0} \sim \lambda_{1}\right] \cup\left[\lambda_{0} \sim \lambda_{2}\right] \cup\left[\lambda_{0} \sim \lambda_{3}\right]$. By Lemma 5.1, $\lambda_{0}=-|c|^{4 / 3}$.

Finally, let $c \neq \pm 3 \sqrt{3}$ belong to $\partial \Omega$. Theorems 4.3 and 4.5 tell us that $\lambda_{1}, \lambda_{2}, \lambda_{3}$ are branch points and that $\lambda_{1}$ has two outgoing arcs and $\lambda_{2}, \lambda_{3}$ have one outgoing arc. If $\Lambda(a)$ had a multiple point, it would be impossible to join the seven arcs without getting new exceptional points or increasing the number of outgoing arcs. It follows that $\Lambda(a)$ has no multiple point and that $\Lambda(a)=\left[\lambda_{1} \sim \lambda_{2}\right] \cup\left[\lambda_{1} \sim \lambda_{3}\right]$.

The two arcs (4.5) emerging for $c \in \partial \Omega \backslash\{ \pm 3 \sqrt{3}\}$ make a cusp at the point $\lambda_{1}$. If $c$ moves along $\partial \Omega$ to $\pm 3 \sqrt{3}$, one of these arcs becomes smaller and smaller, and at $c= \pm 3 \sqrt{3}$ it disappears while the other arc becomes the line segment $[-9,11.25]$. This line segment changes to an analytic arc outside $\Omega$ whereas inside $\Omega$ it transforms into a star with three analytic legs by giving birth to two legs at one of its ends. At $c= \pm i$, the set $\Lambda(a)$ is formed by two arcs making a cusp. As $c$ moves from $\pm i$ into the exterior of $\Omega$, the cusp is bulging out to an analytic curve, and as $c$ moves from $\pm i$ into the interior of $\Omega$, the cusp mutates into a star with three legs by creating a new arc at the cusp point and opening the zero angle of the two arcs of the cusp to a positive angle.

We excluded the parameter $c=0$. Trivially, in that case $\Lambda\left(z^{2}\right)=\{0\}$. If $c \neq 0$ approaches the origin, then the star $\Lambda\left(z^{2}+c z+c z^{-1}\right)$ with the three legs becomes smaller and smaller and eventually it collapses to the point $\{0\}$.

Theorem 6.2. Let $a(z)=z^{2}+c z+c z^{-1}$ with $c \notin \Omega$. Denote the roots of $a^{\prime}(z)=0$ by $t_{1}, t_{2}, t_{3}$ and order them so that $\left|t_{3}\right| \leq\left|t_{2}\right| \leq\left|t_{1}\right|$. Put $\lambda_{k}=$ $a\left(t_{k}\right)$. The limiting set $\Lambda(a)=\left[\lambda_{2} \sim \lambda_{3}\right]$ is a line segment if and only if $c \in(-\infty,-3 \sqrt{3}) \cup(3 \sqrt{3}, \infty)$. In that case $\lambda_{2}$ and $\lambda_{3}$ are real.

Proof. Suppose first that $\pm c \in(3 \sqrt{3}, \infty)$. Such $c$ may be represented in the form (4.2) with $-1 / 2<b<0$. It follows that $w=\left(1+b+b^{2}\right)^{1 / 2}$ may be taken as a positive real number and (4.2) then tells us that all $t_{k}$ are also real numbers. Consequently,

$$
\lambda_{k}=a\left(t_{k}\right)=t_{k}^{2}+c t_{k}+c t_{k}^{-1} \in \mathbb{R} .
$$

The set $\Lambda(a)$ is the intersection of the spectra of the operators $T\left(a_{\varrho}\right)$ for $\varrho \in(0, \infty)$. Since $\overline{a_{\varrho}(\bar{z})}=a_{\varrho}(z)$, the curves $a_{\varrho}(\mathbb{T})$ are symmetric about the real line. Hence so also are the spectra of all $T\left(a_{\varrho}\right)$ and thus also $\Lambda(a)$. But 
an arc starting and ending on $\mathbb{R}$ and being symmetric about $\mathbb{R}$ must be a line segment in $\mathbb{R}$.

Conversely, assume now that $\Lambda(a)=\left[\lambda_{2} \sim \lambda_{3}\right] \subset \mathbb{C}$ is a line segment. Then the tangents to the arc at $\lambda_{2}$ and $\lambda_{3}$ point into opposite directions. By representation (4.4), this means that there is a real number $\mu>0$ such that $a^{\prime \prime}\left(t_{2}\right) t_{2}^{2} /\left(a^{\prime \prime}\left(t_{3}\right) t_{3}^{2}\right)=-\mu$. By the remark at the end of Sect. 4 , we have $a^{\prime \prime}\left(t_{k}\right)=6+2 c / t_{k}$. We may write the numbers $t_{2}, t_{3}, c$ in the form (4.2) with $-1 / 2<\operatorname{Re} b<0$. It follows that

$$
\begin{aligned}
\frac{a^{\prime \prime}\left(t_{2}\right) t_{2}^{2}}{a^{\prime \prime}\left(t_{3}\right) t_{3}^{2}} & =\frac{t_{2}}{t_{3}} \cdot \frac{6 t_{2}+2 c}{6 t_{3}+2 c}=\frac{1}{1+b} \cdot \frac{-3 b-2\left(1+b+b^{2}\right)}{3 b(1+b)-2\left(1+b+b^{2}\right)} \\
& =\frac{1}{1+b} \cdot \frac{2 b^{2}+5 b+2}{b^{2}+b-2}=\frac{1}{1+b} \cdot \frac{(2 b+1)(b+2)}{(b+2)(b-1)} \\
& =-\frac{2 b+1}{1-b^{2}}=-\mu .
\end{aligned}
$$

Put $\mu=1 / \nu$. The equation $(2 b+1) /\left(1-b^{2}\right)=1 / \nu$ is a quadratic equation with the two solutions $b=-\nu \pm \sqrt{\nu^{2}-\nu+1}$. Since $\nu^{2}-\nu+1>0$ for all $\nu$, we conclude that $b$ is a real number. As also $-1 / 2<\operatorname{Re} b<0$, it is only $b \in(-1 / 2,0)$ which can give oppositely directed tangents. But these $b$ correspond just to $\pm c \in(3 \sqrt{3}, \infty)$.

Remark 6.3. The referee again kindly turned attention to the fact that the "if portion" of Theorem 6.2 can also be deduced from the more general result of [11]. Indeed, let $c \in[3 \sqrt{3}, \infty)$. For $x \geq 0$, the function $x^{3}-x$ decreases from 0 to $-2 /(3 \sqrt{3}) \leq-2 / c$ and then increases to infinity. Consequently, there are numbers $0<x_{2}<x_{1}<x_{0}$ such that

$$
\left\{x>0:-2 / c \leq x^{3}-x \leq 2 / c\right\}=\left(0, x_{2}\right] \cup\left[x_{1}, x_{0}\right] .
$$

It follows that the set $\left\{r>0:-2 / c \leq 1 / r^{3}-1 / r \leq 2 / c\right\}$ is of the form $\left[r_{0}, r_{1}\right] \cup\left[r_{2}, \infty\right)$ with $r_{j}=1 / x_{j}$. Consider the curve

$$
K=\left\{\kappa(r):=\sigma(r) \pm i \tau(r): r \in\left[r_{0}, r_{1}\right]\right\}
$$

with

$$
\sigma(r)=\frac{c}{2} \frac{1-r^{2}}{r^{2}}, \quad \tau(r)=\frac{1}{2 r^{2}} \sqrt{4 r^{3}-c^{2}\left(1-r^{2}\right)^{2}} .
$$

Note that $\tau(r)>0$ for $r \in\left(r_{0}, r_{1}\right)$ and that $\tau\left(r_{0}\right)=\tau\left(r_{1}\right)=0$. This reveals that $K$ is a Jordan curve. We have $\sigma^{2}+\tau^{2}=r^{2}$, and hence $\kappa=\kappa(r)$ satisfies the quadratic equation

$$
\kappa^{2}-\frac{c\left(1-r^{2}\right)}{r^{2}} \kappa+r^{2}=0 .
$$

Dividing by $\kappa r^{2}$ we get

$$
\kappa / r^{2}-\frac{c\left(1-r^{2}\right)}{r^{4}}+1 / \kappa=0 .
$$

The last two equations imply

$$
f(\kappa)=\kappa^{2}+c \kappa+c / \kappa=\frac{c\left(1-r^{2}\right)}{r^{2}} \kappa-r^{2}+c \kappa+\frac{c^{2}\left(1-r^{2}\right)}{r^{4}}-\frac{c \kappa}{r^{2}}
$$




$$
=\frac{c^{2}\left(1-r^{2}\right)}{r^{4}}-r^{2} \in \mathbb{R},
$$

showing that $f(K) \subset \mathbb{R}$ and hence, by [11], proving that $\Lambda(f)$ is a real line segment.

\section{Acknowledgements}

We sincerely thank the referee for several useful comments and in particular for contributing Remarks 4.6 and 6.3, which nicely show how a general result works in concrete situations.

Funding Open Access funding enabled and organized by Projekt DEAL.

Open Access. This article is licensed under a Creative Commons Attribution 4.0 International License, which permits use, sharing, adaptation, distribution and reproduction in any medium or format, as long as you give appropriate credit to the original author(s) and the source, provide a link to the Creative Commons licence, and indicate if changes were made. The images or other third party material in this article are included in the article's Creative Commons licence, unless indicated otherwise in a credit line to the material. If material is not included in the article's Creative Commons licence and your intended use is not permitted by statutory regulation or exceeds the permitted use, you will need to obtain permission directly from the copyright holder. To view a copy of this licence, visit http:// creativecommons.org/licenses/by/4.0/.

Publisher's Note Springer Nature remains neutral with regard to jurisdictional claims in published maps and institutional affiliations.

\section{References}

[1] Beam, R.M., Warming, R.F.: The asymptotic spectra of banded Toeplitz and quasi-Toeplitz matrices. SIAM J. Sci. Comput. 14, 971-1006 (1993)

[2] Böttcher, A., Grudsky, S.M.: Spectral Properties of Banded Toeplitz Matrices. SIAM, Philadelphia, PA (2005)

[3] Böttcher, A., Silbermann, B.: Introduction to Large Truncated Toeplitz Matrices. Universitext. Springer, New York (1999)

[4] Böttcher, A., Widom, H.: Two remarks on spectral approximations for WienerHopf operators. J. Integral Equations Appl. 6, 31-36 (1994)

[5] Duits, M., Kuijlaars, A.B.J.: An equilibrium problem for the limiting eigenvalue distribution of banded Toeplitz matrices. SIAM J. Matrix Anal. Appl. 30, 173196 (2008)

[6] Garoni, C., Serra-Capizzano, S.: Generalized Locally Toeplitz Sequences: Theory and Applications, vol. I. Springer, Cham (2017)

[7] Hirschmann Jr., I.I.: The spectra of certain Toeplitz matrices. Illinois J. Math. 11, 145-159 (1967)

[8] Nikolski, N.: Toeplitz Matrices and Operators. Cambridge University Presss, Cambridge (2020) 
[9] Reichel, L., Trefethen, L.N.: Eigenvalues and pseudo-eigenvalues of Toeplitz matrices. Linear Algebra Appl. 162/164, 153-185 (1992)

[10] Schmidt, P., Spitzer, F.: The Toeplitz matrices of an arbitrary Laurent polynomial. Math. Scand. 8, 15-38 (1960)

[11] Shapiro, B., Štampach, F.: Non-self-adjoint Toeplitz matrices whose principal submatrices have real spectrum. Constr. Approx. 49, 191-226 (2019)

[12] Szegö, G.: Ein Grenzwertsatz über die Toeplitzschen Determinanten einer reellen positiven Funktion. Math. Ann. 76, 490-503 (1915)

[13] Trefethen, L.N., Embree, M.: Spectra and Pseudospectra. The Behavior of Nonnormal Matrices and Operators. Princeton University Press, Princeton, NJ (2005)

[14] Tyrtyshnikov, E.E.: A unifying approach to some old and new theorems on distribution and clustering. Linear Algebra Appl. 232, 1-43 (1996)

[15] Tyrtyshnikov, E.E., Zamarashkin, N.L.: Distribution of the eigenvalues and singular numbers of Toeplitz matrices under weakened requirements on the generating function. Sb. Math. 188, 1191-1201 (1997)

[16] Tyrtyshnikov, E.E., Zamarashkin, N.L.: Toeplitz eigenvalues for Radon measures. Linear Algebra Appl. 343/344, 345-354 (2002)

[17] Ullman, J.L.: A problem of Schmidt and Spitzer. Bull. Am. Math. Soc. 73, 883-885 (1967)

[18] Widom, H.: Eigenvalue distribution of nonselfadjoint Toeplitz matrices and the asymptotics of Toeplitz determinants in the case of nonvanishing index. Oper. Theory Adv. Appl. 48, 387-421 (1990)

[19] Widom, H.: Eigenvalue distribution for nonselfadjoint Toeplitz matrices. Oper. Theory Adv. Appl. 71, 1-8 (1994)

\author{
Albrecht Böttcher $(\bowtie)$ \\ Fakultät für Mathematik, TU Chemnitz \\ Chemnitz 09107 \\ Germany \\ e-mail: aboettch@mathematik.tu-chemnitz.de \\ Juanita Gasca \\ Departamento de Matemáticas \\ CINVESTAV del I.P.N. \\ 07360 Ciudad de México \\ Mexico \\ e-mail: jgasca@math.cinvestav.mx
}




\section{Sergei M. Grudsky}

Departamento de Matemáticas

CINVESTAV del I.P.N.

07360 Ciudad de México

Mexico

and

Regional Mathematical Center of the Southern Federal University

Rostov-on-Don

Russia 344007

e-mail: grudsky@math.cinvestav.mx

Anatoli V. Kozak

Regional Mathematical Center of the Southern Federal University

Rostov-on-Don

Russia 344007

e-mail: avkozak@bmail.ru

Received: September 4, 2020.

Revised: December 11, 2020. 